\title{
Progenitor-derived human endothelial cells evade alloimmunity by CRISPR/ Cas9-mediated complete ablation of MHC expression
}

\author{
Jonathan Merola, ${ }^{1}$ Melanie Reschke, ${ }^{2}$ Richard W. Pierce, ${ }^{3}$ Lingfeng Qin, ${ }^{1}$ Susann Spindler, ${ }^{1}$ \\ Tania Baltazar, ${ }^{4}$ Thomas D. Manes, ${ }^{4}$ Francesc Lopez-Giraldez, ${ }^{5}$ Guangxin Li, ${ }^{1}$ Laura C. Bracaglia, ${ }^{2}$ \\ Catherine Xie, ${ }^{4}$ Nancy Kirkiles-Smith, ${ }^{4}$ W. Mark Saltzman, ${ }^{2}$ Gregory T. Tietjen, ${ }^{1}$ George Tellides, ${ }^{1}$ \\ and Jordan S. Pober ${ }^{4}$ \\ 'Department of Surgery, Yale School of Medicine, New Haven, Connecticut, USA. ²Department of Biomedical Engineering, \\ Yale School of Engineering and Applied Science, New Haven, Connecticut, USA. ${ }^{3}$ Department of Pediatrics, and \\ ${ }^{4}$ Department of Immunobiology, Yale School of Medicine, New Haven, Connecticut, USA. ${ }^{5}$ Yale Center for Genome Analysis \\ and Department of Genetics, Yale University, New Haven, Connecticut, USA.
}

\begin{abstract}
Tissue engineering may address organ shortages currently limiting clinical transplantation. Off-theshelf engineered vascularized organs will likely use allogeneic endothelial cells (ECs) to construct microvessels required for graft perfusion. Vasculogenic ECs can be differentiated from committed progenitors (human endothelial colony-forming cells or HECFCs) without risk of mutation or teratoma formation associated with reprogrammed stem cells. Like other ECs, these cells can express both class I and class II major histocompatibility complex (MHC) molecules, bind donorspecific antibody (DSA), activate alloreactive T effector memory cells, and initiate rejection in the absence of donor leukocytes. CRISPR/Cas9-mediated dual ablation of $\beta_{2}$-microglobulin and class II transactivator (CIITA) in HECFC-derived ECs eliminates both class I and II MHC expression while retaining EC functions and vasculogenic potential. Importantly, dually ablated ECs no longer bind human DSA or activate allogeneic $\mathrm{CD} 4^{+}$effector memory $\mathrm{T}$ cells and are resistant to killing by $\mathrm{CD} 8^{+}$ alloreactive cytotoxic T lymphocytes in vitro and in vivo. Despite absent class I MHC molecules, these ECs do not activate or elicit cytotoxic activity from allogeneic natural killer cells. These data suggest that HECFC-derived ECs lacking MHC molecule expression can be utilized for engineering vascularized grafts that evade allorejection.
\end{abstract}

Conflict of interest: The authors have declared that no conflict of interest exists.

Copyright: () 2019, American Society for Clinical Investigation.

Submitted: May 1, 2019 Accepted: September 11, 2019 Published: October 17, 2019

Reference information: /CI Insight. 2019;4(20):e129739. https://doi.org/10.1172/jci. insight.129739.

\section{Introduction}

Organ transplantation is the most effective therapy for patients afflicted with end-stage failure of the heart, lungs, kidney, liver, or intestine, but its current application is significantly limited by the lack of available allografts (1-4). Over 110,000 patients are on transplant waiting lists, while only 35,000 organ transplants occur annually in the US (5). Bioengineered replacement organs have the potential to address this shortage. Endothelial cell-lined (EC-lined) microvessels will be required for adequate perfusion of the engineered donor tissue. Circulating endothelial colony-forming cells (ECFCs), which are abundant in umbilical cord blood, display substantially greater replicative potential than vessel wall-derived ECs and readily differentiate into functional ECs with capacity to self-organize into microvessels (6). As committed progenitors, ECFCs do not differentiate into other cell types, retain normal chromosome number, and unlike induced pluripotent stem cells do not give rise to teratomas (7). These attributes make ECFCs an attractive cell source for constructing vascularized engineered tissues (8).

Critically ill patients may need off-the-shelf replacement organs preassembled using cells from allogeneic sources (9). Responses to allogeneic natural organs can provide important insights as to how recipient immune systems will respond. Broadly, transplant rejection may be precipitated by donor-reactive antibodies or host $\mathrm{T}$ cell-mediated responses. Nearly $15 \%$ of transplant candidates on the waiting list harbor circulating antibodies reactive with non-self allelic forms of class I and class II major histocompatibility 
complex (MHC) molecules (designated as panel-reactive antibodies [PRAs]), capable of precipitating graft rejection $(10,11)$. Graft recipients may also develop donor-specific antibodies (DSAs) de novo after transplantation, most often reactive with class II MHC molecules on graft ECs (12). Binding of PRAs or DSAs to ECs leads to the activation of complement and deposition of membrane attack complexes (MACs) on the EC surface. MACs do not cause EC lysis but instead trigger proinflammatory signaling cascades that enhance recruitment and activation of alloreactive $T$ cells promoting rejection (13). Of clinical significance, the presence of a DSA is highly correlated with late graft loss (14).

$\mathrm{T}$ cell-mediated rejection most frequently occurs within the first posttransplant year and is initiated by effector memory $\mathrm{T}$ (Tem) cells that recognize graft $\mathrm{MHC}$ molecules, each complexed to one of a large number of different peptides $(15,16)$. Recognition of graft MHC molecules on human ECs by circulating Tem cells induces transendothelial recruitment. Infiltrating $\mathrm{CD}^{+}$Tem cells differentiate into cytotoxic $\mathrm{T}$ lymphocytes (CTLs) and mediate acute rejection $(17,18)$. CTL differentiation from CD $8^{+}$Tem cells is aided by activated $\mathrm{CD}^{+}$Tem cells; these cells release interleukin-2 (IL-2) and other mediators, which promote the survival and expansion of CTLs (19). Collectively, graft class I and II MHC molecules on ECs serve as targets of alloantibody and T cell-mediated recognition that precipitate rejection responses. Importantly, ECs in an engineering tissue may represent the only graft cell type capable of initiating cellular rejection (20).

Since the expression of MHC molecules is a necessary property of ECs to trigger both humoral and cellular rejection, loss of MHC expression should diminish the capacity of ECs to cause these processes. We previously used clustered regularly interspaced palindromic repeats and its associated nuclease CRISPR-associated protein 9 (CRISPR/Cas9), to generate stable biallelic gene disruption in ECs derived from ECFCs that could then be clonally expanded (21). CRISPR/Cas9 targeting of the class II transactivator (CIITA) in ECs eliminated class II MHC expression and significantly diminished activation of $\mathrm{CD}^{+}$memory T cells (19). However, CIITA ${ }^{\text {null }}$ ECs were only partially protected from vascular destruction, as cytotoxicity is principally mediated by $\mathrm{CD} 8^{+}$CTLs recognizing class I MHC. Here, we extended this approach to target $\beta_{2}$-microglobulin, an invariant component of the class I MHC complex, both alone and in combination with CIITA. We find that dual CRISPR/Cas9 targeting of $\beta_{2}$-microglobulin and CIITA eliminates class I and II MHC expression in human ECs derived from ECFCs while retaining core EC functions, including the capacity to form microvessels. As expected, dually ablated class I and class II MHC-null ECs exposed to high-titer PRA sera show markedly reduced antibody binding and markedly diminished capacity to activate allogeneic $\mathrm{CD}^{+}$and $\mathrm{CD} 4^{+}$Tem cell proliferative responses. Despite loss of class I MHC molecule expression, $\beta_{2}$-microglobulin ${ }^{\text {null }}$ ECs do not show susceptibility to allogeneic natural killer (NK) cells. Furthermore, tissue-engineered and perfused microvessels generated from $\beta_{2}$-microglobulin ${ }^{\text {null }}+$ CIITA $^{\text {null }}$ ECs in immunodeficient mouse hosts are resistant to destruction by adoptively transferred allogeneic (to the ECs) peripheral blood mononuclear cells (PBMCs) in vivo. These data suggest that tissue-engineered constructs perfused through vessels lined by allogeneic ECs lacking MHC molecules will be significantly less prone to rejection.

\section{Results}

CRISPR/Cas9-mediated genetic targeting of $\beta_{2}$-microglobulin and CIITA in human ECs. We previously described a method for high-efficiency biallelic genomic disruption of the CIITA gene locus in ECFC-derived ECs (21). Resultant EC clonal populations were unable to express class II MHC molecules following human interferon $\gamma$ (IFN- $\gamma$ ) treatment (21). To evaluate the effects of ablating class I MHC expression, we targeted $\beta_{2}$-microglobulin, an invariant component of the class I MHC complex. We targeted exonic regions of the (i) $\beta_{2}$-microglobulin locus alone, (ii) CIITA locus alone, (iii) both $\beta_{2}$-microglobulin and CIITA loci, or (iv) the adeno-associated viral integration site 1 (AAVS1) locus, using the latter as a control target that would not affect class I or II MHC expression. Modified cultures were treated with human IFN- $\gamma$ for 72 hours to both maximize class I MHC expression and restore class II MHC expression, reversing downmodulation associated with cell culture. Flow cytometry was used to sort viable cells lacking class I and/or II MHC expression, which represented $40 \%-50 \%$ of the population in cells targeted for a single locus and $5 \%-20 \%$ in dually ablated $\beta_{2}$-microglobulin ${ }^{\text {null }}+$ CIITA $^{\text {null }}$ ECs (Supplemental Figure 1; supplemental material available online with this article; https://doi.org/10.1172/jci.insight.129739DS1). Subsequent re-treatment with IFN- $\gamma$ failed to re-induce class I and HLA-DR expression in $\beta_{2}$-microglobulin- and CIITA-targeted cells, respectively. Dually targeted cells similarly showed absence of both class I MHC and HLA-DR following IFN- $\gamma$ treatment. Control cells transduced with an irrelevant guide strand vector targeting AAVS1 
expressed high levels of class I MHC and HLA-DR, the predominant class II locus expressed in ECs (Figure 1A). Further characterization of surface and total MHC protein expression after IFN- $\gamma$ treatment, as assayed by intracellular staining and flow cytometry, revealed an absence of both class I MHC components in cells targeted for $\beta_{2}$-microglobulin ablation (Figure 1B) and all 3 human class II loci in cells in which CIITA was targeted (Figure 1C). Single-cell-derived colonies were expanded from ECs transduced with guide strands targeting the $\beta_{2}$-microglobulin and/or CIITA loci; genomic DNA from each expanded colony was then isolated. Sequencing of polymerase chain reaction (PCR) amplicons obtained from the regions surrounding the targeted protospacer-adjacent motif at both $\beta_{2}$-microglobulin and CIITA exonic loci confirmed biallelic mutation (Figure 1D).

Transplanted hepatocytes derived from $\beta_{2}$-microglobulin ${ }^{\text {null }}$ mice into wild-type recipients demonstrated their capacity to endocytose exogenous $\beta_{2}$-microglobulin and reconstitute class I MHC in trans (22). We therefore evaluated the ability of $\beta_{2}$-microglobulin ${ }^{\text {null }}$ human ECs to reconstitute class I MHC expression after culture with human serum, with or without recombinant $\beta_{2}$-microglobulin protein supplementation and observed no rescue of class I MHC expression in $\beta_{2}$-microglobulin ${ }^{\text {null }}$ ECs under these conditions (Supplemental Figure 2A). $\beta_{2}$-Microglobulin normally pairs with class I heavy chains during the process of peptide loading in the endoplasmic reticulum (ER). In the absence of $\beta_{2}$-microglobulin, accumulation of unpaired class I heavy chains could potentially trigger an unfolded protein response. We evaluated this possibility by assessing expression of CCAAT-enhancer-binding protein homologous protein (CHOP), a major transcription factor of the ER stress response. We observed no significant expression of CHOP in control or $\beta_{2}$-microglobulin ${ }^{\text {null }}$ cells (Supplemental Figure $2 \mathrm{~B}$ ). CHOP was readily detected following treatment of cells with thapsigargin, a positive control for induction of ER stress.

RNA sequencing and functional characterization of CRISPR/Cas9-modified ECs. RNA sequencing was performed to compare the transcriptome of cells targeted with a control guide RNA (AAVS1) to $\beta_{2}$-microglobulin $^{\text {null }}$, CIITA ${ }^{\text {null }}$, and dually ablated $\beta_{2}$-microglobulin ${ }^{\text {null }}+$ CIITA $^{\text {null }}$ ECs following treatment with IFN- $\gamma$. Differential gene expression analysis revealed 57 significantly differentially expressed genes (FDR-adjusted $P<0.05$ ) with a fold change of 4 or higher when comparing $\beta_{2}$-microglobulin ${ }^{\text {null }}$, CIITA $^{\text {null }}$, and dually ablated $\beta_{2}$-microglobulin ${ }^{\text {null }}+$ CIITA $^{\text {null }}$ ECs with the control ECs (AAVS1) (Tables 1 and 2). As expected, loss of known CIITA-regulated genes were significantly underrepresented in cells ablated of CIITA (Fisher's exact test $P<0.0001$ ) when compared with the AAVS1-targeted EC control (23). Only 1 gene, encoding the elastin microfibril interface-locate protein 1 (EMILIN1), was significantly downregulated in $\beta_{2}$-microglobulin ${ }^{\text {null }}$ ECs compared with the AAVS1-targeted EC control. This gene was also downregulated in both CIITA ${ }^{\text {null }}$ and dually ablated $\beta_{2}$-microglobulin ${ }^{\text {null }}+$ CIITA $^{\text {null }}$ ECs. EMILIN1 expression was similar in control guide strand-treated cells and untransduced cells, suggesting that its downregulation is not a general feature of lentiviral transduction or of Cas9 activity despite being caused by totally unrelated guide strands in the MHC-targeted cells. The gene encoding the EMILIN1 protein is found on chromosome 2 in humans, not physically linked to the locations of genes encoding $\beta_{2}$-microglobulin (chromosome 15) or CIITA (chromosome 16) and knowledge of its expression and function in ECs is unknown.

Several functional phenotypic characteristics were compared between ECs edited with a control guide RNA (AAVS1), $\beta_{2}$-microglobulin ${ }^{\text {null }}$ ECs, CITA ${ }^{\text {null }} \mathrm{ECs}$, and combined $\beta_{2}$-microglobulin $^{\text {null }}+$ CIITA $^{\text {null }}$ ECs. All 4 EC types exhibited similar morphology and junctional staining patterns of PECAM-1 (CD31) and VE-cadherin (CD144), as evaluated by confocal microscopy (Figure 2A). Moreover, monolayer cultures of all 4 EC types formed junctions with comparable barriers (Figure 2B) that were similarly disrupted after treatment with thrombin or tumor necrosis factor $\alpha(\mathrm{TNF}-\alpha)$, as assessed by electrical cell impedance sensing (Figure 2C). All 4 EC types normally upregulated the adhesion molecules ICAM-1 and PD-L1 in response to treatment with IFN- $\gamma$, indicative of preserved activation responses (Figure 2D). These attributes suggest that $\beta_{2}$-microglobulin ${ }^{\text {null }}+$ CIITA $^{\text {null }}$ ECs retained core endothelial phenotypic functions in vitro.

ECs will self-assemble into perfusable vessels, an attribute critical for their use in tissue engineering. To assess if this ability was retained in our Cas9-modified ECs, control (AAVS1), $\beta_{2}$-microglobulin ${ }^{\text {null }}$, and dually ablated $\beta_{2}$-microglobulin ${ }^{\text {null }}+$ CIITA $^{\text {null }}$ ECs were suspended in a collagen/fibronectin protein gel matrix and implanted subcutaneously in the abdominal wall of C.B-17 severe combined immunodeficient/beige (SCID/bg) mice. Two weeks later, the animals were injected with human IFN- $\gamma$ every other day for 5 days to induce MHC molecule re-expression $(17,18)$. Mice were sacrificed and collagen gels were harvested 15 minutes after intravenous injection of fluorescently labeled Ulex europeanus agglutinin (UEA), which is reactive with human blood group $\mathrm{H}$ antigen expressed on all human ECs. Fluorescence 

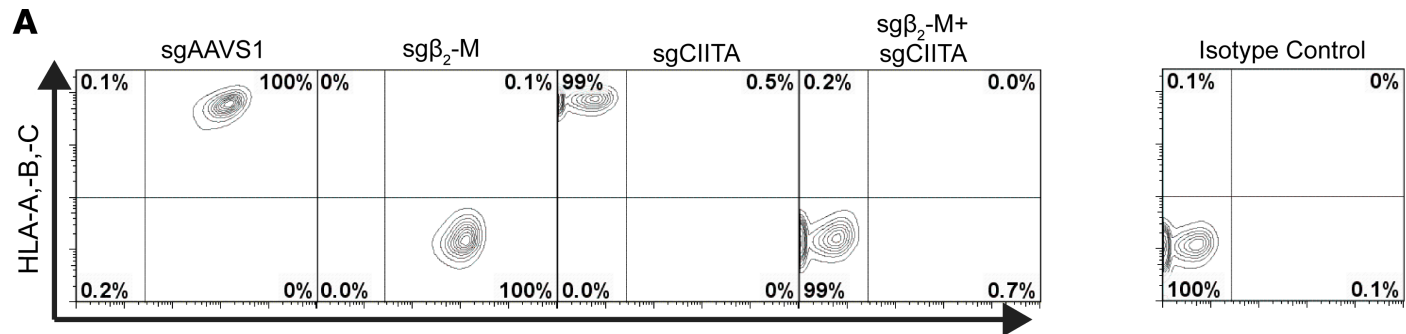

HLA-DR

B
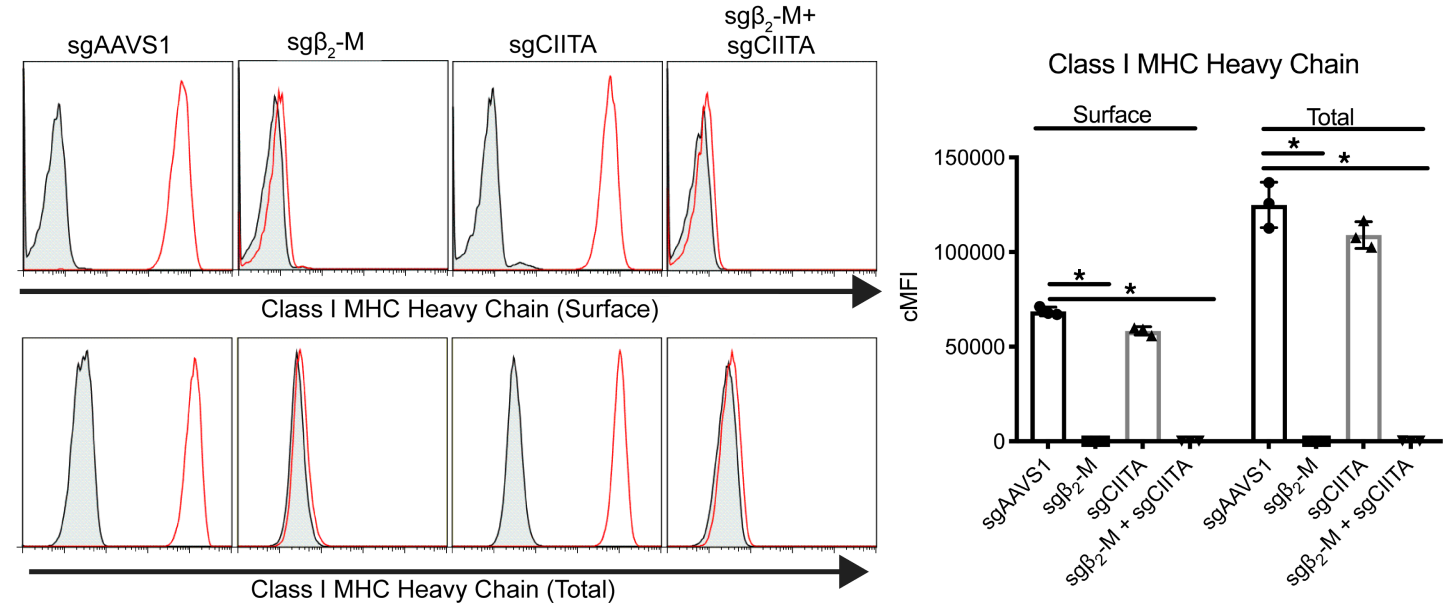

C

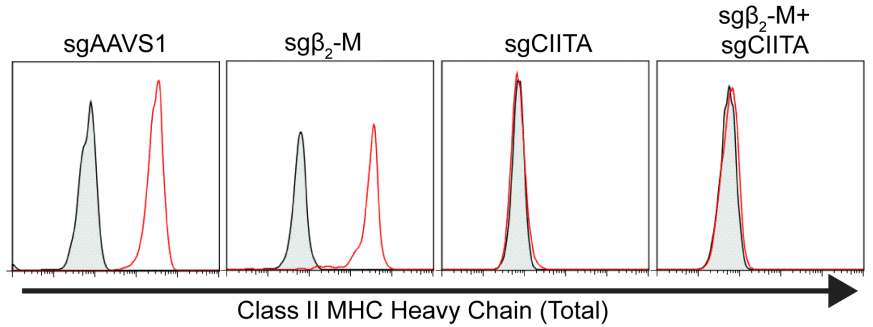

Class II MHC Heavy Chain
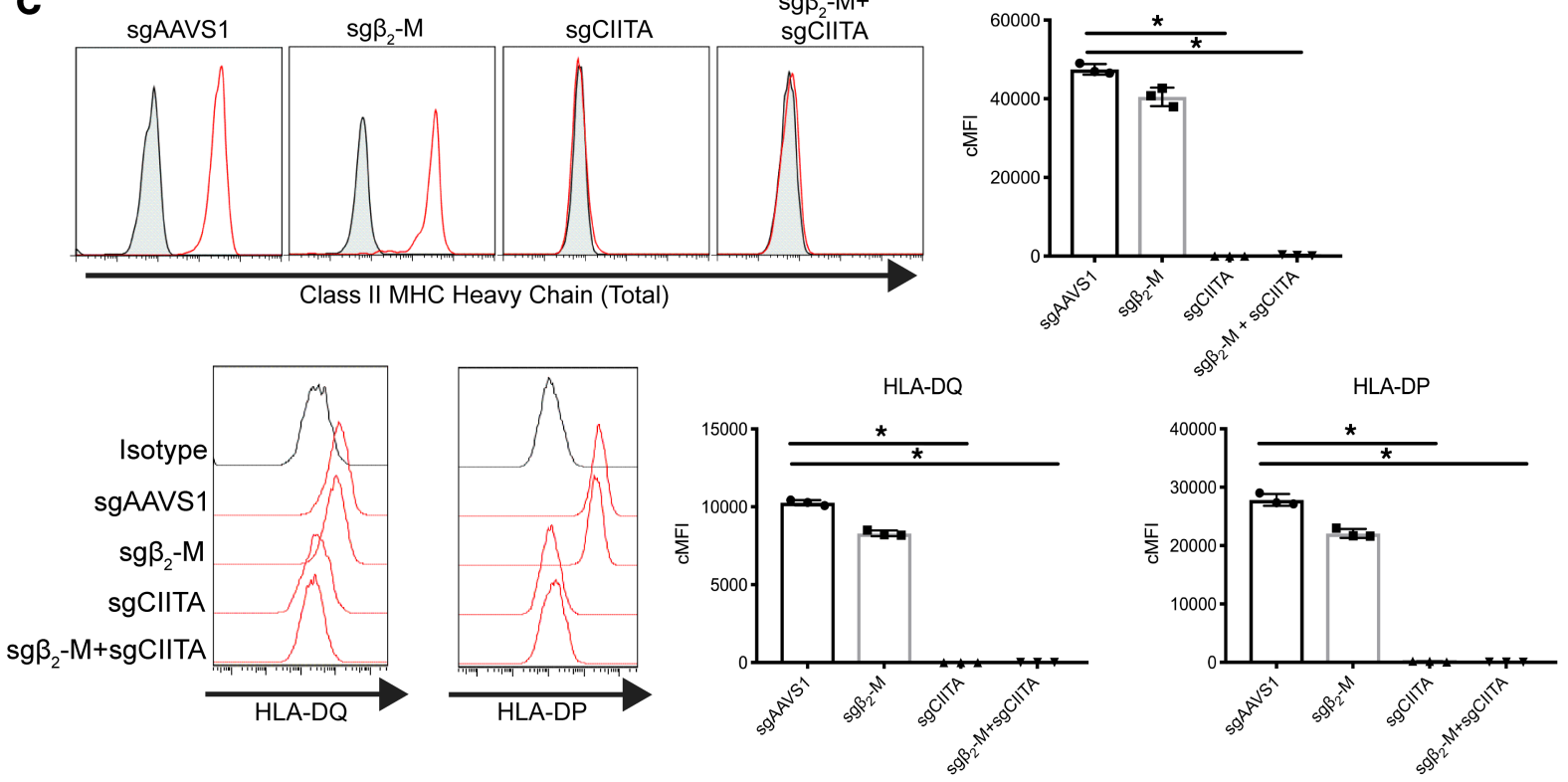

D

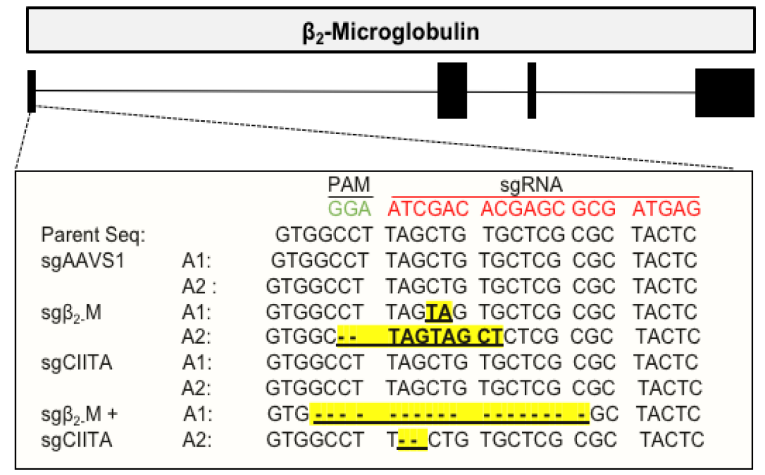

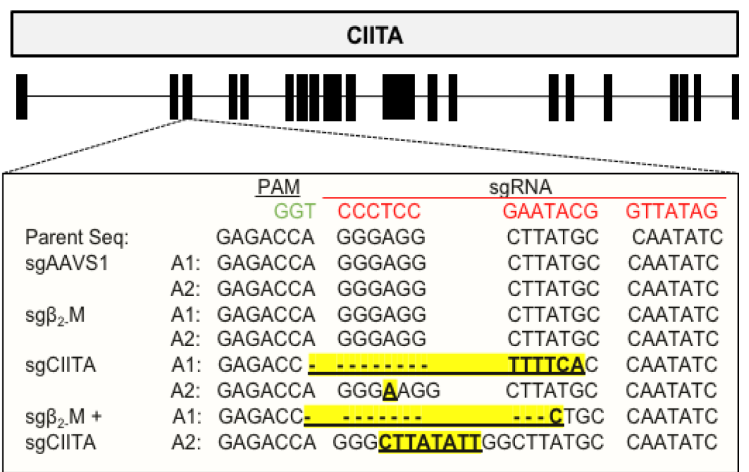


Figure 1. CRISPR/Cas9 biallelic disruption of $\beta_{2}$-microglobulin and CIITA eliminates class I and II MHC expression in human cord-blood endothelial cells. (A) Clonally expanded colonies of sorted Cas9-modified ECFCs transduced with guide strands targeting $\beta_{2}$-microglobulin, CIITA, or a control gene locus (AAVS1). (B) Surface class I MHC heavy chain and total class I MHC heavy chain assayed by surface and intracellular flow cytometry reveals absence of expression in $\beta_{2}$-microglobulin null ECFCs. (C) Total class II MHC heavy chain, HLA-DQ, and HLA-DP expression is absent in CIITA ${ }^{\text {null }}$ ECFCs. (D) Biallelic indel mutations noted in $\beta_{2}$-microglobulin and CIITA gene loci in cells transduced with targeting guide strands. Representative plots shown from 3 unrelated donors shown as mean \pm SEM.

microscopy revealed formation of perfused (UEA ${ }^{+}$) human EC-lined vessels in all 3 groups. Control ECs showed expression of both class I and class II MHC molecules. Similarly to the in vitro behavior, no class I MHC expression was detectable in $\beta_{2}$-microglobulin ${ }^{\text {null }}$ cells and neither class I nor II MHC molecules were expressed by dually ablated $\beta_{2}$-microglobulin ${ }^{\text {null }}+$ CIITA $^{\text {null }}$ ECs (Figure $2 E$ ). Thus, MHC-ablated ECs retain core endothelial characteristics in vivo, making them useful for tissue engineering.

Alloantibody-mediated responses of CRISPR/Cas9-modified ECS. Circulating antibodies recognizing nonself class I and II MHC molecules that predispose patients to rejection of allogeneic grafts are present in pre-sensitized transplant candidates ( $15 \%$ of waitlist candidates) $(10,11)$. Some of these individuals have high titers of PRA, recognizing over $80 \%$ of all tested HLA specificities. Binding of alloantibody to graft ECs triggers complement activation and deposition of terminal complement MACs on EC membranes. Biopsies from such rejecting grafts often show uninjured ECs despite the presence of bound antibody and MACs $(24,25)$. In cell culture and in humanized mouse models, MACs are endocytosed, transferred to endosomes, and trigger noncanonical nuclear factor- $\mathrm{kB}(\mathrm{NF}-\mathrm{\kappa B})$ signaling (13). Sera pooled from highly sensitized patients (high-PRA serum) were added to control vector-transduced, $\beta_{2}$-microglobulin ${ }^{\text {null }}$, CIITA $^{\text {null }}$, and dually ablated $\beta_{2}$-microglobulin ${ }^{\text {null }}+$ CIITA $^{\text {null }}$ ECs under conditions that support complement activation. Compared with control ECs, $\beta_{2}$-microglobulin ${ }^{\text {null }}$ and CIITA ${ }^{\text {null }}$ cells exhibited a reduction in alloantibody binding (Figure 3A), complement deposition (Figure 3B), and complement-mediated noncanonical NF-kB signaling (Figure 3C). Dually ablated $\beta_{2}$-microglobulin ${ }^{\text {null }}+C$ CITA ${ }^{\text {null }}$ ECFCs showed nearly complete elimination of alloantibody binding and complement deposition. Signaling through the complement-dependent noncanonical NF-kB pathway was markedly diminished but not completely absent. Residual signaling may have been induced by cytokines present in the circulation of some individuals or by binding of alloantibody reactive with targets other than MHC molecules (26).

$T$ cell responses to CRISPR/Cas9-modified ECS. Human allografts can be rejected when $C D 8^{+}$and/or $\mathrm{CD}^{+}$circulating Tem cells recognize non-self class I and II MHC molecules expressed on graft ECs $(16,27)$. We therefore tested the ability of IFN- $\gamma$-treated control vector-transduced, $\beta_{2}$-microglobulin ${ }^{\text {null }}$, $\mathrm{CIITA}^{\text {null }}$, and dually ablated $\beta_{2}$-microglobulin ${ }^{\text {null }}+\mathrm{CIITA}^{\text {null }} \mathrm{ECs}$ to activate allogeneic $\mathrm{CD} 8^{+}$and $\mathrm{CD} 4^{+} \mathrm{Tem}$ cells in vitro. As expected, IFN- $\gamma$-pretreated control ECs induce proliferation in a measurable subpopulation of allogeneic $\mathrm{CD} 4^{+}$and $\mathrm{CD} 8^{+}$Tem cells. Compared with control cocultures, $\mathrm{CD} 8^{+}$Tem cells showed a marked reduction in proliferation when cocultured with $\beta_{2}$-microglobulin ${ }^{\text {null }}$ and $\beta_{2}$-microglobulin ${ }^{\text {null }}+\mathrm{CI}$ ITA $^{\text {null }}$ cells. Similarly, a lack of proliferation of $\mathrm{CD} 4^{+}$Tem cells was noted when cocultured with CIITA ${ }^{\text {null }}$ and $\beta_{2}$-microglobulin ${ }^{\text {null }}+$ CIITA $^{\text {null }}$ cells (Figure $4 A$ ). Proliferating CD4 ${ }^{+}$Tem cells cocultured with class II MHC-expressing ECs expressed the activation marker HLA-DR. However, this activation marker was not expressed when $C D 4^{+}$Tem cells were cocultured with $\mathrm{CIITA}^{\text {null }}$ and $\beta_{2}$-microglobulin ${ }^{\text {null }}+\mathrm{CIITA}^{\text {null }}$ cells (Figure 4B). Thus, the absence of MHC molecules on ECs prevents TCR-mediated activation and expansion of alloreactive $\mathrm{T}$ cells.

Cell-mediated rejection of allografts is largely mediated by CTL clones that recognize different peptides complexed with non-self allelic forms of class I MHC molecules expressed on target cells. To evaluate whether the absence of class I MHC was sufficient to abrogate CTL-mediated cytotoxicity, alloreactive $\mathrm{T}$ cell clones were raised on wild-type ECs $(17,18)$ and subsequently cocultured with control vector-transduced, $\beta_{2}$-microglobulin ${ }^{\text {null }}$, CIITA ${ }^{\text {null }}$, or dually ablated $\beta_{2}$-microglobulin ${ }^{\text {null }}+$ CIITA $^{\text {null }} E C s$ from the same donor. Absence of class I MHC was sufficient to nearly eliminate T cell-mediated killing in vitro (Figure $4 C$ ).

NK cell responses to CRISPR/Cas9-modified ECS. NK cells are innate lymphoid cells that use the same mechanisms to kill target cells as CTLs. Almost all NK cells express an activating low-affinity receptor for certain IgG isotypes and thus kill target cells to which relevant IgG isotypes are bound. NK cells can also kill targets when activated by cytokines or by engagement of activating receptors with target-cell ligands. However, many NK cells also express inhibitory receptors that recognize certain self-allelic forms of HLA-B and -C 
Table 1. Genes significantly upregulated with CRISPR/Cas9 targeting in ECs

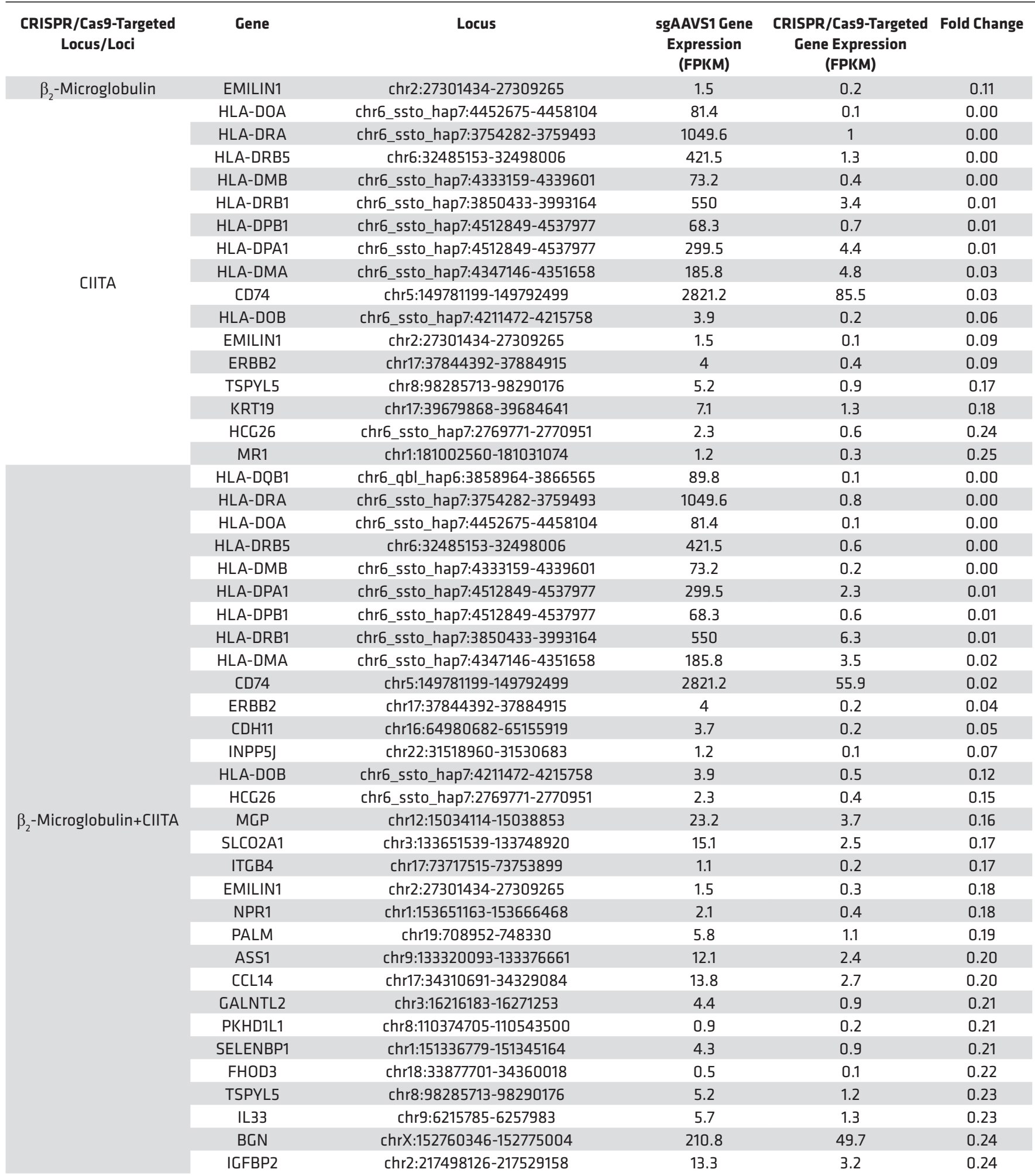

Genes significantly upregulated (FDR-adjusted $P<0.05$ ) with CRISPR/Cas9 targeting compared with control vector (AAVS1) targeting in ECs with fold change $>4$ after stimulation with IFN- $\gamma(50 \mathrm{ng} / \mathrm{mL})$ for 96 hours. Expression values are fragments per kilobase of exon per million fragments mapped (FPKM). 
Table 2. Genes significantly downregulated with CRISPR/Cas9 targeting in ECs

\begin{tabular}{|c|c|c|c|c|c|}
\hline $\begin{array}{c}\text { CRISPR/Cas9-Targeted } \\
\text { Locus/Loci }\end{array}$ & Gene & Locus & $\begin{array}{l}\text { sgAAVS1 Gene } \\
\text { Expression } \\
\text { (FPKM) }\end{array}$ & $\begin{array}{c}\text { CRISPR/Cas9- } \\
\text { Targeted Gene } \\
\text { Expression (FPKM) }\end{array}$ & Fold Change \\
\hline \multirow{8}{*}{$\beta_{2}$-Microglobulin } & NCAM2 & chr21:22370632-22912517 & 0.1 & 1 & 12.9 \\
\hline & SCUBE1 & chr22:43599228-43739394 & 0.1 & 1.2 & 10.9 \\
\hline & TNNT1 & chr19:55644160-55660606 & 0.1 & 1.4 & 10.2 \\
\hline & POU4F1 & chr13:79173229-79177695 & 0.1 & 0.6 & 9.5 \\
\hline & HOXB9 & chr17:46698518-46703835 & 0.2 & 1.6 & 7.2 \\
\hline & GREM1 & chr15:33010204-33026870 & 0.2 & 1.7 & 7 \\
\hline & PAPPA & chr9:118916070-119164600 & 0.3 & 1.2 & 4.8 \\
\hline & EEF1A2 & chr20:62119365-62130505 & 0.6 & 2.4 & 4.1 \\
\hline \multirow{5}{*}{ CIITA } & COL6A2 & chr21:47518032-47552763 & 0.2 & 3.8 & 18.6 \\
\hline & SIM1 & chr6:100836749-100911551 & 0 & 0.6 & 14.5 \\
\hline & NCAM2 & chr21:22370632-22912517 & 0.1 & 0.9 & 10.7 \\
\hline & TAF7L & chrX:100523240-100548059 & 0.1 & 0.6 & 10.6 \\
\hline & GREM1 & chr15:33010204-33026870 & 0.2 & 2.2 & 9.2 \\
\hline \multirow{9}{*}{$\beta_{2}$-Microglobulin+CIITA } & MMP20 & chr11:102447565-102496063 & 0 & 0.4 & $\infty$ \\
\hline & LARGE & chr22:33669061-34316416 & 0 & 1.1 & 28.7 \\
\hline & PAPPA2 & chr1:176432306-176811970 & 0 & 0.8 & 23.8 \\
\hline & POU4F1 & chr13:79173229-79177695 & 0.1 & 0.5 & 8.1 \\
\hline & SERPINB2 & chr18:61554938-61571124 & 1.1 & 8.5 & 7.7 \\
\hline & AFF3 & chr2:100163715-100759037 & 0.4 & 3.1 & 7.6 \\
\hline & FAM129A & chr1:184760165-184943682 & 1.9 & 10 & 5.2 \\
\hline & LPXN & chr11:58294343-58345639 & 7.1 & 34.7 & 4.9 \\
\hline & $\mathrm{KIT}$ & chr4:55524094-55606881 & 0.2 & 0.8 & 4.2 \\
\hline
\end{tabular}

Genes significantly downregulated (FDR-adjusted $P<0.05$ ) with CRISPR/Cas9 targeting compared with control vector (AAVS1) targeting in ECs with fold change $>4$ after stimulation with IFN- $\gamma(50 \mathrm{ng} / \mathrm{mL})$ for 96 hours. Expression values are fragments per kilobase of exon per million fragments mapped (FPKM).

molecules, and lack of class I MHC molecules on allogeneic hematopoietic cells or some tumor cells results in NK cell activation and killing (28). Since class I MHC molecules will be absent on $\beta_{2}$-microglobulin ${ }^{\text {null }}$ and $\beta_{2}$-microglobulin ${ }^{\text {null }}+$ CIITA $^{\text {null }}$ ECs, we examined if the absence of class I MHC on human ECs conferred greater capacity for $\mathrm{NK}$ activation by culturing allogeneic $\mathrm{CD} 3{ }^{-} \mathrm{CD} 56^{+} \mathrm{CD} 335^{+} \mathrm{CD} 16^{+} \mathrm{NK}$ cells on $\beta_{2}$-microglobulin $^{\text {null }}$ and $\beta_{2}$-microglobulin ${ }^{\text {null }}+$ CIITA $^{\text {null }}$ ECs as compared with cocultures with control ECs or the K562 leukemic cell line, a known NK cell target used as a positive control. Release of TNF- $\alpha$ and IFN- $\gamma$ as well as cytotoxicity mediated by human NK cells was observed when cultured with K562 cells. However, no enhancement of cytokine release was detected in class I-absent cells compared with control ECs (Figure $5 \mathrm{~A}$ ) and no significant NK-mediated cytolysis was observed upon coculture with $\beta_{2}$-microglobulin ${ }^{\text {null }}$ ECs compared with control, despite an intact capacity of NK cells to lyse ECs bound by alloantibody (Figure 5B). Therefore, the absence of MHC I on ECs is not sufficient to trigger NK-mediated activation and cytolysis.

Failure to activate allogeneic NK cells could result from absence of activating ligands on ECs. To investigate this further, the transcriptional abundance of known NK cell ligands was evaluated using RNA sequencing data obtained previously (Table 1). From these we identified expression of HLA-E, MICA/MICB, CD111 (nectin-1), CD112 (nectin-2), and CD155 (PVR). We then compared ECFC-derived ECs as well as adult human dermal microvascular ECs (HDMECs) under both resting conditions and following 48 hours of treatment with IFN- $\gamma$ or TNF- $\alpha$ to simulate inflammatory conditions. K562 cells, known NK-cell targets, were included as a comparator. As shown in Figure 5C, both ECFCs and HDMECs had similar responses to these proinflammatory cytokines, exhibiting increased levels of HLA-E, CD111, CD112, and CD155 compared with K562 cells, while HLA-G was highly expressed by K562 cells but not the ECs. The function of these NK cell ligands may be either activating or inhibitory, depending on their receptor engagement. For example, CD112 may be activating when engaging with DNAM-1, but inhibitory when engaging with TIGIT $(29,30)$. Similarly, HLA-E may activate NK cells when engaging with NKG2C and inhibitory when binding NKG2A (31). Thus, our expression data indicate that while ECs express some potentially activating NK ligands, they are not sufficient for ECs to activate NK cells in the absence of MHC I. 

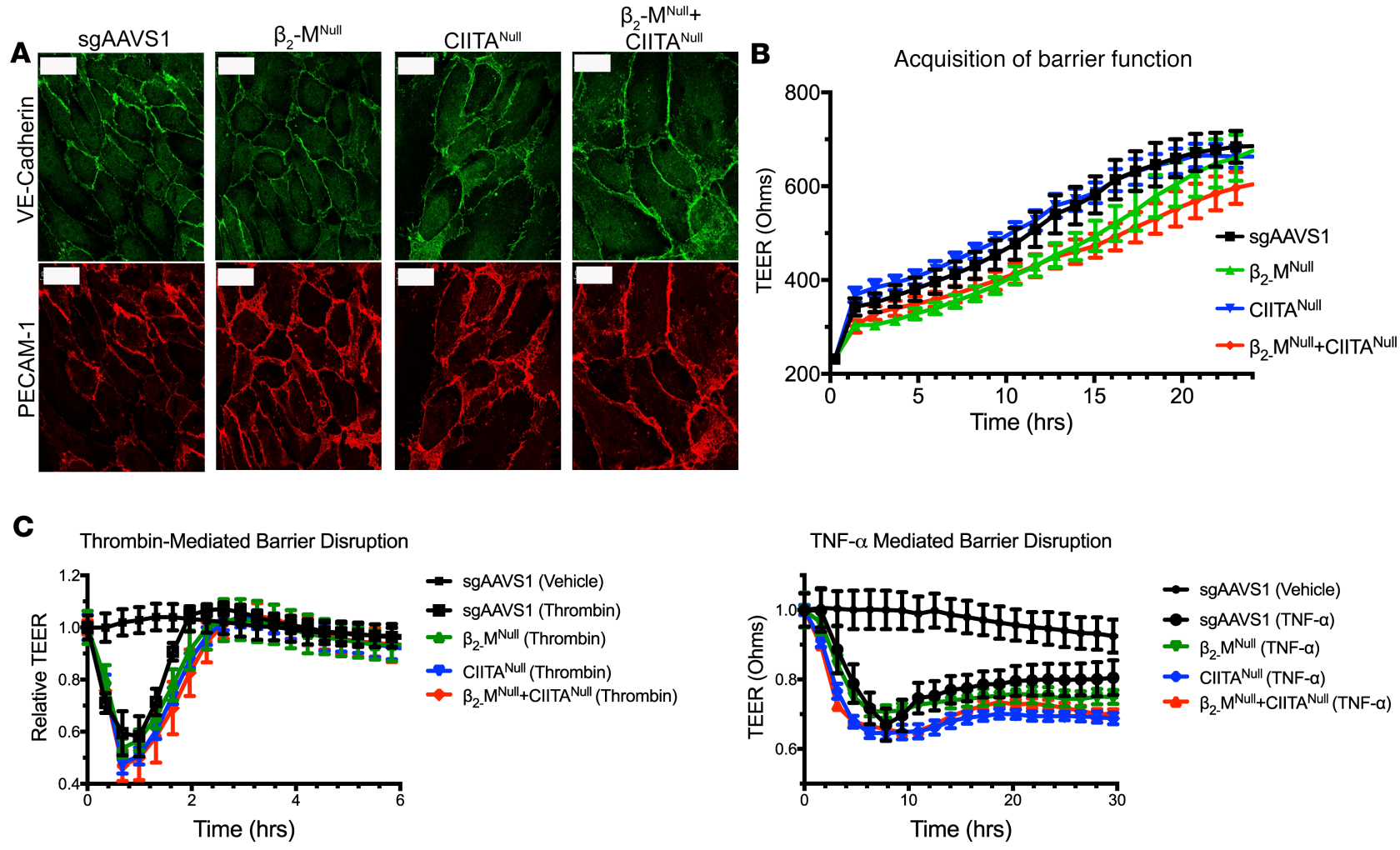

D
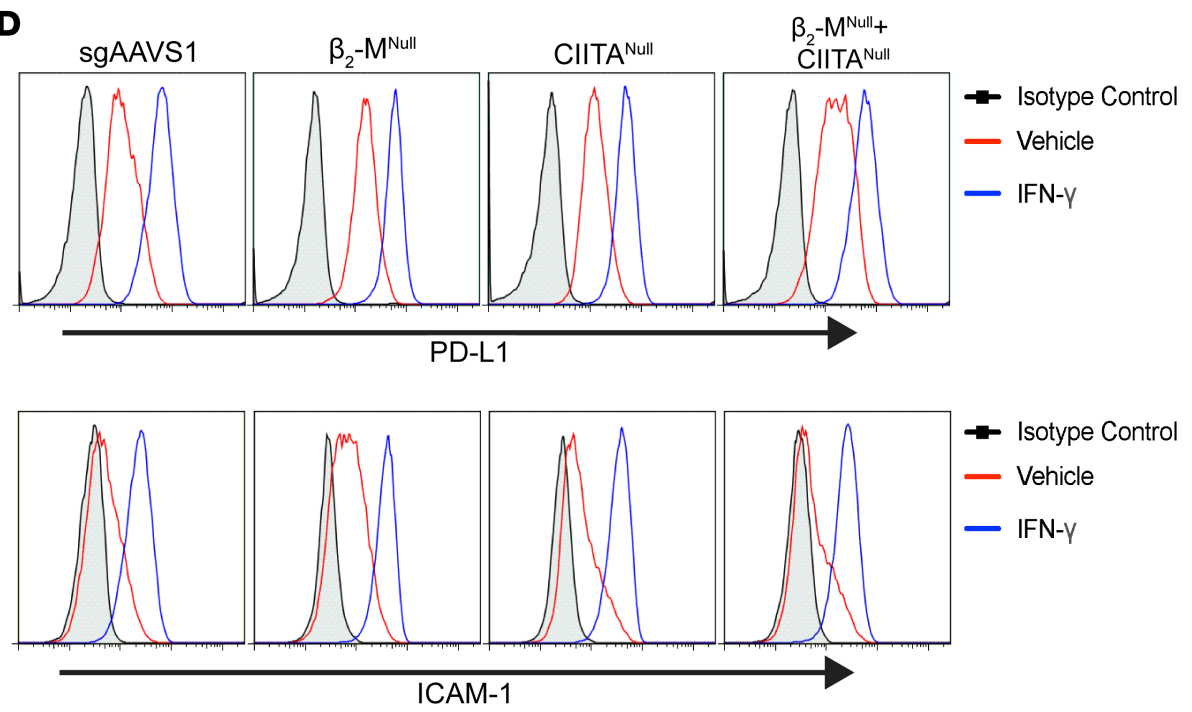

E

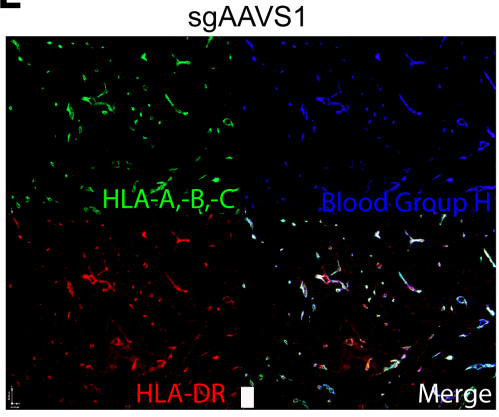

$\beta_{2}-M^{\text {Null }}$

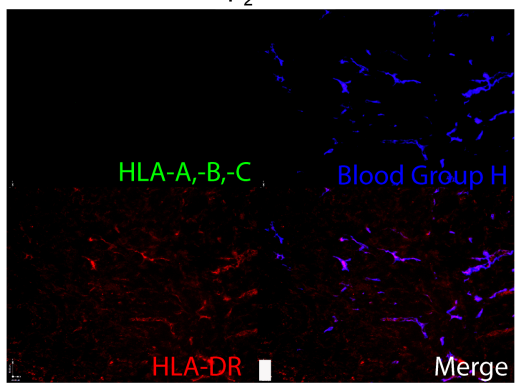

Figure 2. CRISPR/Cas9 ablation of $\beta_{2}$-microglobulin and CIITA does not alter core endothelial cell functional characteristics. (A) Confocal microscopy exhibiting similar junctional VE-cadherin (CD144) and PECAM-1 (CD31) staining and cell morphology. Scale bars: $20 \mu \mathrm{m}$. (B) Formation of equivalent barriers over time as measured by transendothelial electrical resistance (TEER). (C) Barriers are equally disrupted in response to thrombin ( $1 \mathrm{U} / \mathrm{L})$ and TNF- $\alpha(10 \mathrm{ng} / \mathrm{mL})$. (D) Upregulation of ICAM-1 and PD-L1 in response to IFN- $\gamma(50 \mathrm{ng} / \mathrm{mL}$, 48 hours) in MHC-ablated compared with control (AAVS1) ECs. (E) Vessel formation preserving preimplantation MHC expression in Cas9-modifed ECs 2 weeks following suspension in collagen/fibronectin gel and implantation in an immunodeficient mouse in vivo. Scale bars: $50 \mu \mathrm{m}$. Representative of 4 independent donors.

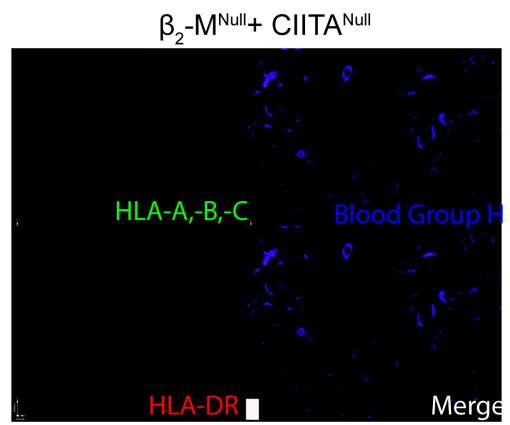


A

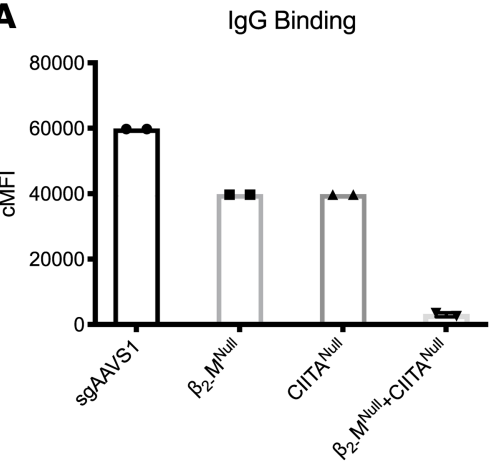

B

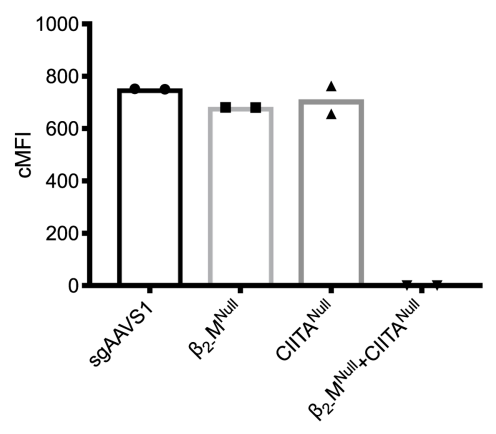

$c$
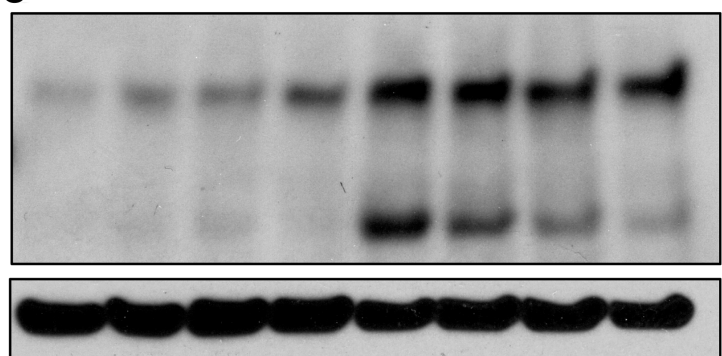

ß-Actin

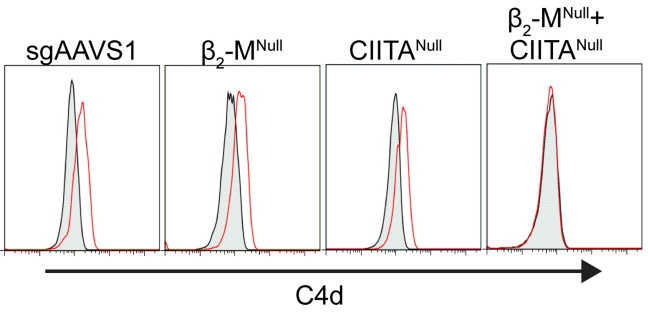

Unsensitized 25\% Human ABO Serum

$25 \%$ High cPRA Serum

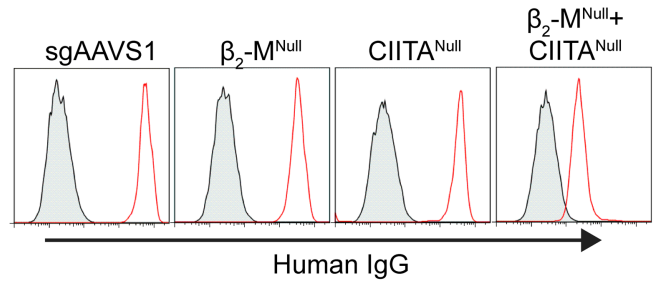

Isotype control

$\square 25 \%$ High cPRA Serum p52

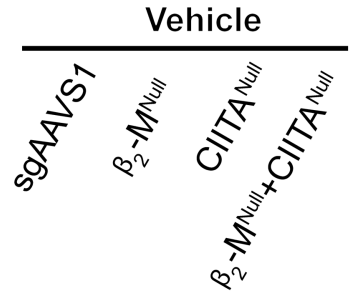

$25 \%$ High PRA Serum
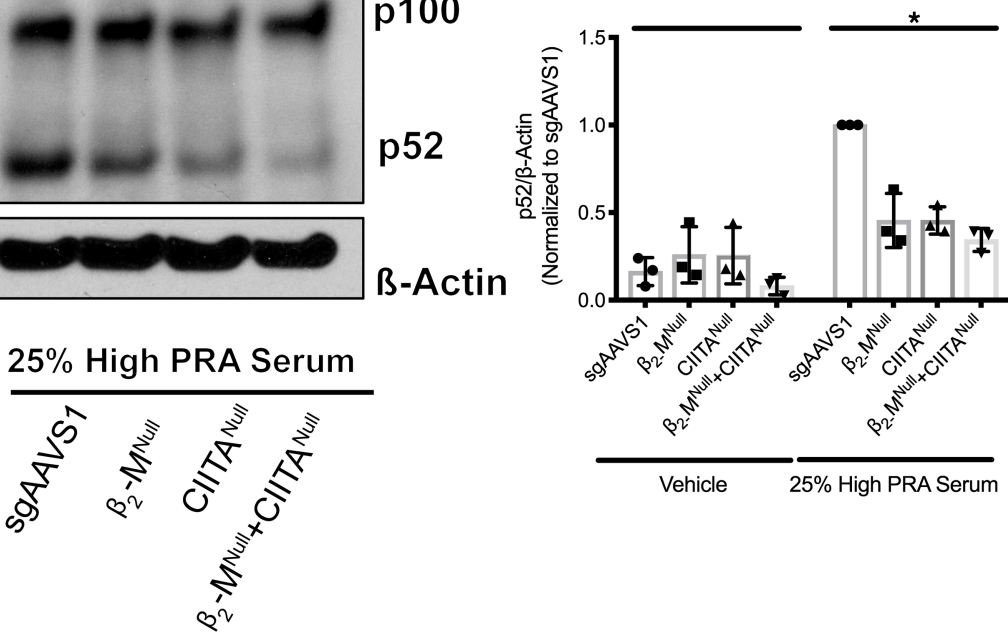

Figure 3. $\beta_{2}$-Microglobulin ${ }^{\text {null }}+$ CIITA ${ }^{\text {null }}$ ECs evade alloantibody-mediated binding, complement deposition, and complement-dependent NF-KB signaling. Cas9-modified ECs were cocultured with $25 \%$ high-PRA serum in gelatin veronal buffer for 2 hours and surface (A) human IgG and (B) human C4d were assessed by flow cytometry (2 donors). (C) Immunoblot of Cas9-modified ECs cocultured with $25 \%$ high-PRA serum shows diminished noncanonical NF- $\mathrm{KB}$ signaling, characterized by processing of $p 100$ to $p 52$, in $\beta_{2}$-microglobulin ${ }^{\text {null }}+C$ IITTA $A^{\text {null }}$ ECs compared with control (AAVS1) $E C s$. Densitometry exhibiting mean \pm SEM relative $p 52 / \beta$-actin expression of 3 independent endothelial donors and high-titer cPRA pooled serum pairs run in triplicate.

To determine whether NK cells are simply ignorant of or transiently inhibited by ECs or if they are rendered anergic by their interactions with ECs, we cocultured isolated NK cells with control (sgAAVS1) ECs, class I-knockout ( $\beta_{2}$-microglobulinnull) ECs, or no-target cells, harvested them 6 hours later, and then added them back to K562 NK-cell targets to assess if they could still be activated. Remarkably, preincubation of NK cells with $\beta_{2}$-microglobulin ${ }^{\text {null }}$ ECs but not control ECs prevents IFN- $\gamma$ release in the 
CD4

A
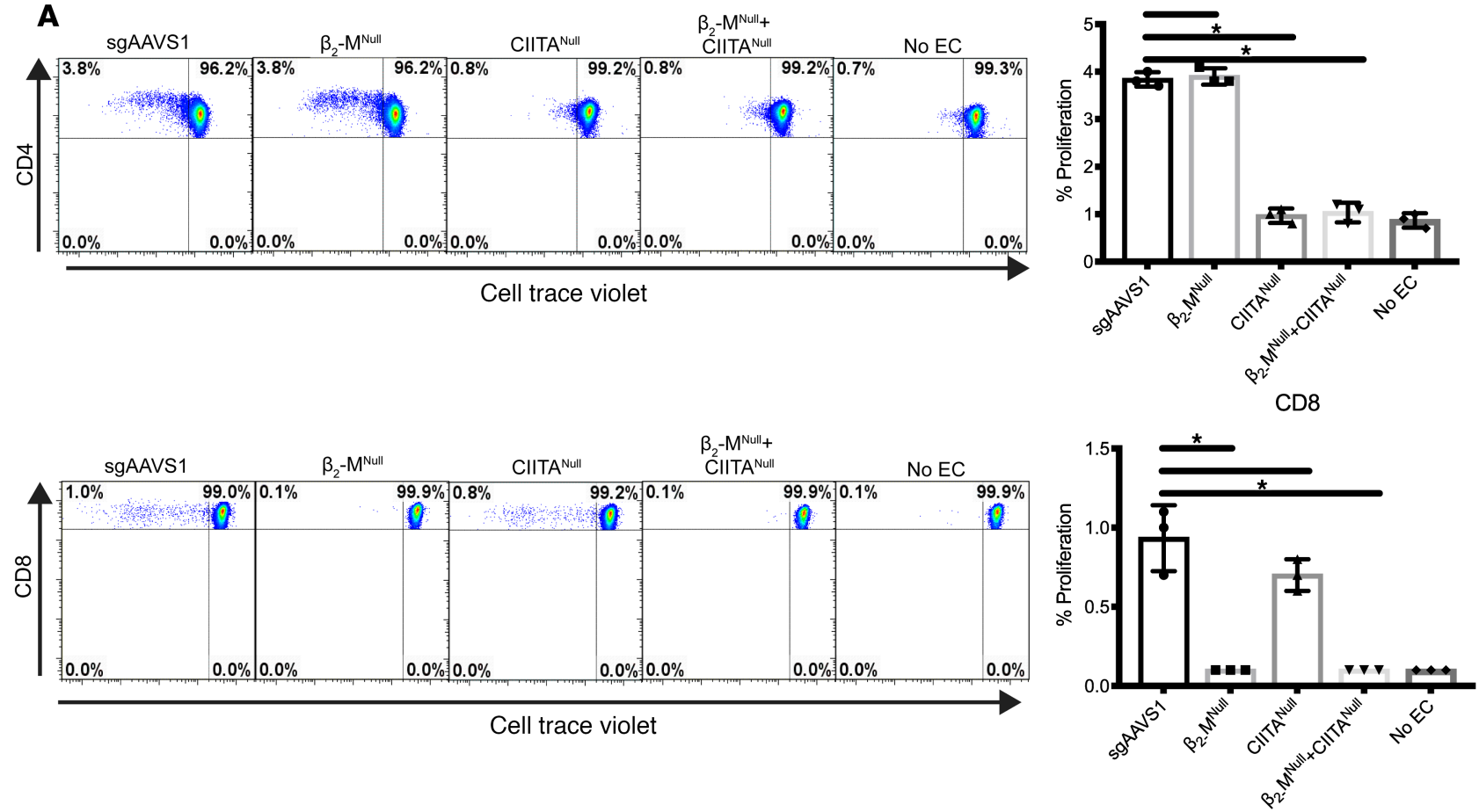

B

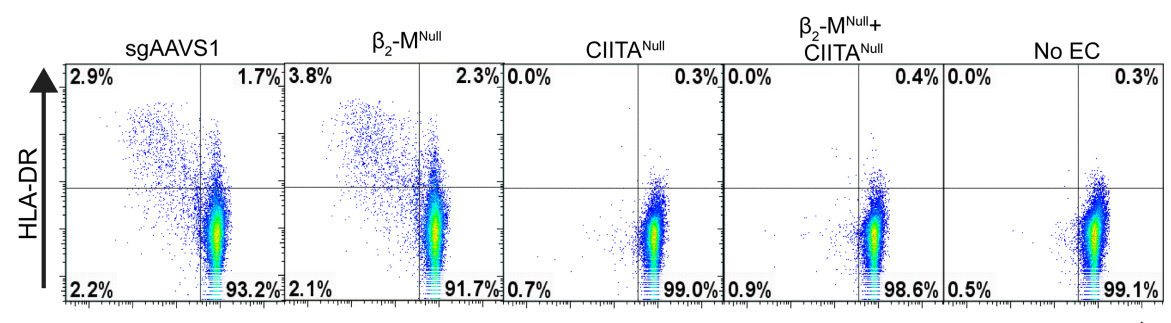

Cell trace violet

Activation of $\mathrm{CD}^{+}$Cells

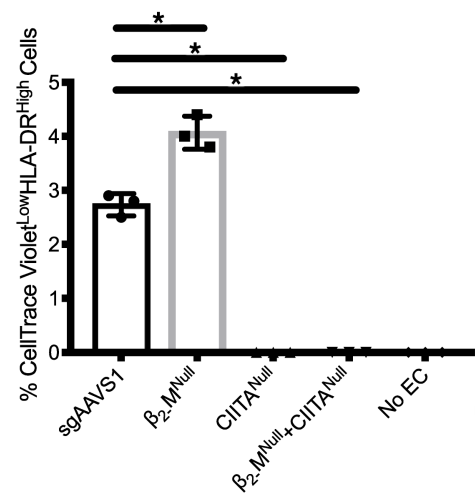

- sgAAVS1
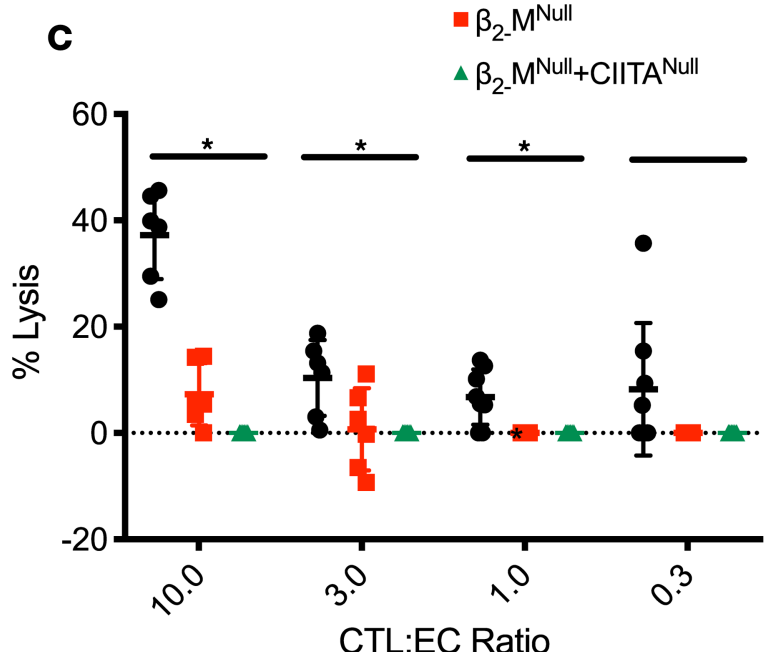

Figure 4. Ablation of class I and II MHC expression abrogates CD4 and $\mathrm{CD}^{+} \mathrm{T}^{\mathrm{T}}$ cell proliferation, $\mathrm{CD4} 4^{+}$activation, and $\mathrm{CD8} 8^{+}$cytotoxicity. (A) CIITA ${ }^{\text {null }}$ and $\beta_{2}$-microglobulin ${ }^{\text {null }}+$ CIITA $^{\text {null }} E C$ s exhibit a reduction in $C D 4^{+}$Tem cell proliferation and $\beta_{2}$-microglobulin ${ }^{\text {null }}$ and $\beta_{2}$-microglobulin ${ }^{\text {null }}+$ CIITA $^{\text {null }} E$ ECs exhibit a reduction in CD8 ${ }^{+}$Tem cell proliferation compared with control (sgAAVS1) ECs. (B) CD4+ Tem cells cocultured with CIITA ${ }^{\text {null }} E C s$ fail to express activation markers. (C) Cytotoxic T lymphocytes raised on donor-matched wild-type ECs fail to kill $\beta_{2}$-microglobulin ${ }^{\text {null }}$ ECs compared with control ECs, as measured by calcein AM release. Representative of 3 independent donor pairs shown as mean \pm SEM. 
A

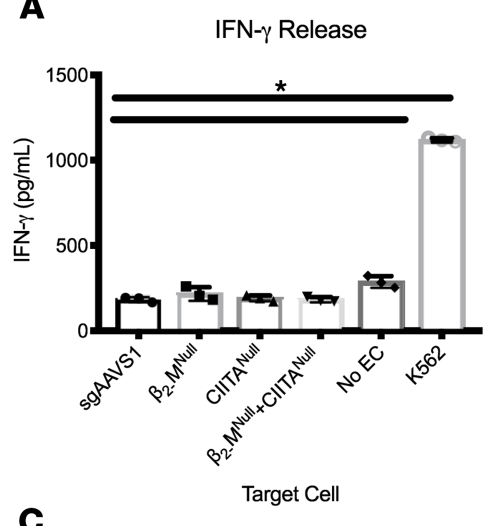

C

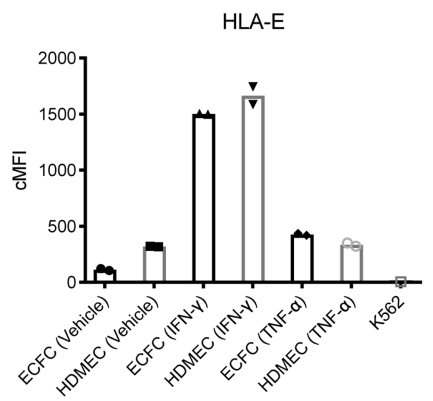

CD111

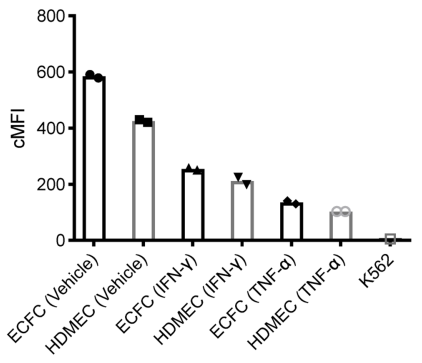

PD-L1

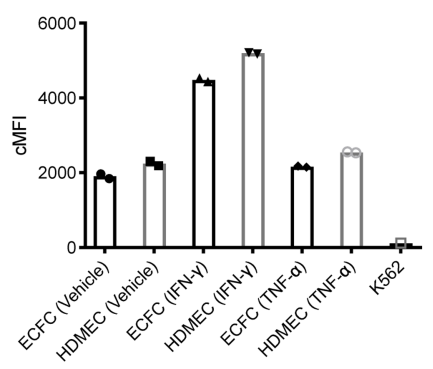

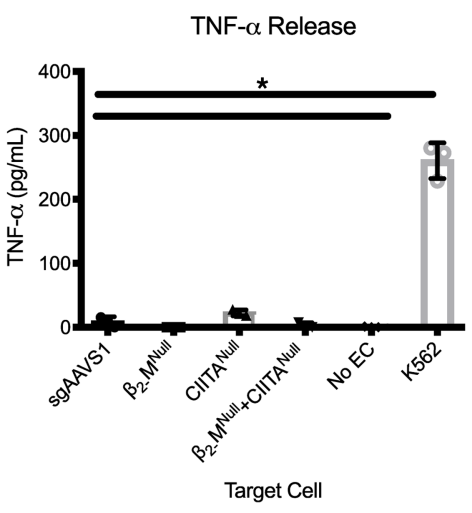

HLA-G

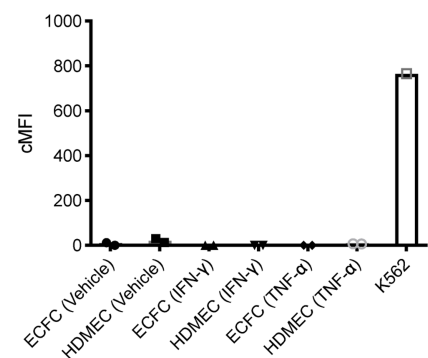

CD112

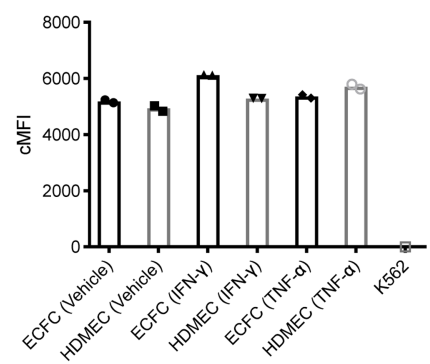

VCAM-1

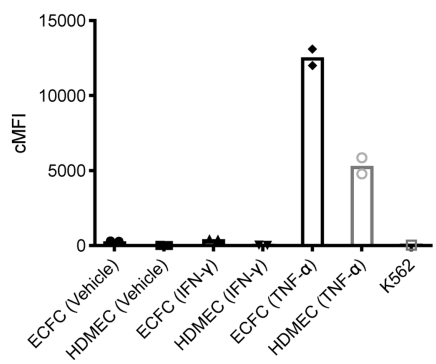

D

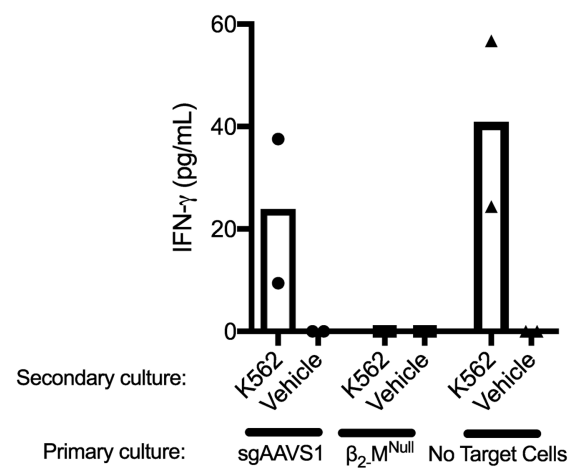

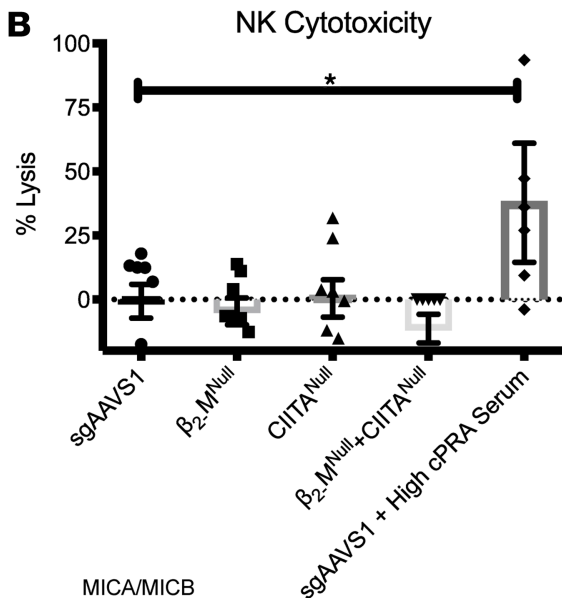

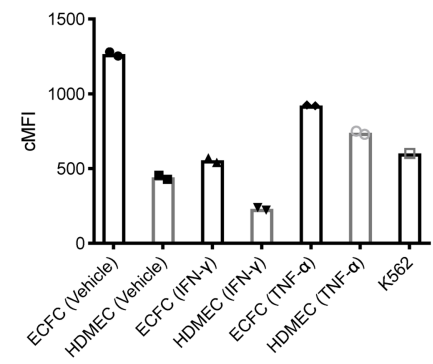

CD155

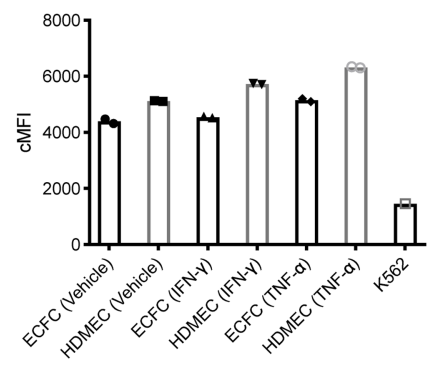

Figure 5. Absence of class I MHC on human endothelium does not enhance NK cell activation or cytotoxicity. (A) IFN- $\gamma$ and TNF- $\alpha$ ELISA of supernatant in cocultures of human NK cells with MHC-absent ECs. No enhancement of cytokine release was observed in $\beta_{2}$-microglobulin ${ }^{\text {null }}$ cells compared with control ECs. (B) Cytotoxicity measured by calcein AM release, with human alloantibody-bound ECs included as a positive control. Representative of 4 independent donor pairs (mean \pm SEM). (C) Expression of NK cell ligands in human cord blood-derived ECs (ECFCs), adult human dermal microvascular endothelial cells (HDMECs), and leukemic K562 NK cell targets under resting conditions and 48 hours of IFN- $\gamma$ or TNF- $\alpha$. VCAM- 1 and PD-L1 expression are included as control antigens known to be upregulated in ECs in the presence of TNF- $\alpha$ or IFN- $\gamma$, respectively. Representative of 2 independent donors. (D) NK cell release of IFN- $\gamma$ on secondary exposure of $K 562$ cells following 6 hours of primary coculture with control (sgAAVS1), $\beta_{2}$-microglobulin $^{\text {null }}$, or no-target cells. Absence of IFN- $\gamma$ release on exposure to $\mathrm{K} 562$ cells following $\beta_{2}$-microglobulin ${ }^{\text {null }} E C$ coculture suggests $\beta_{2}$-microglobulin ${ }^{\text {null }} E C s$ induce NK cell anergy. Representative of 2 independent donors. 

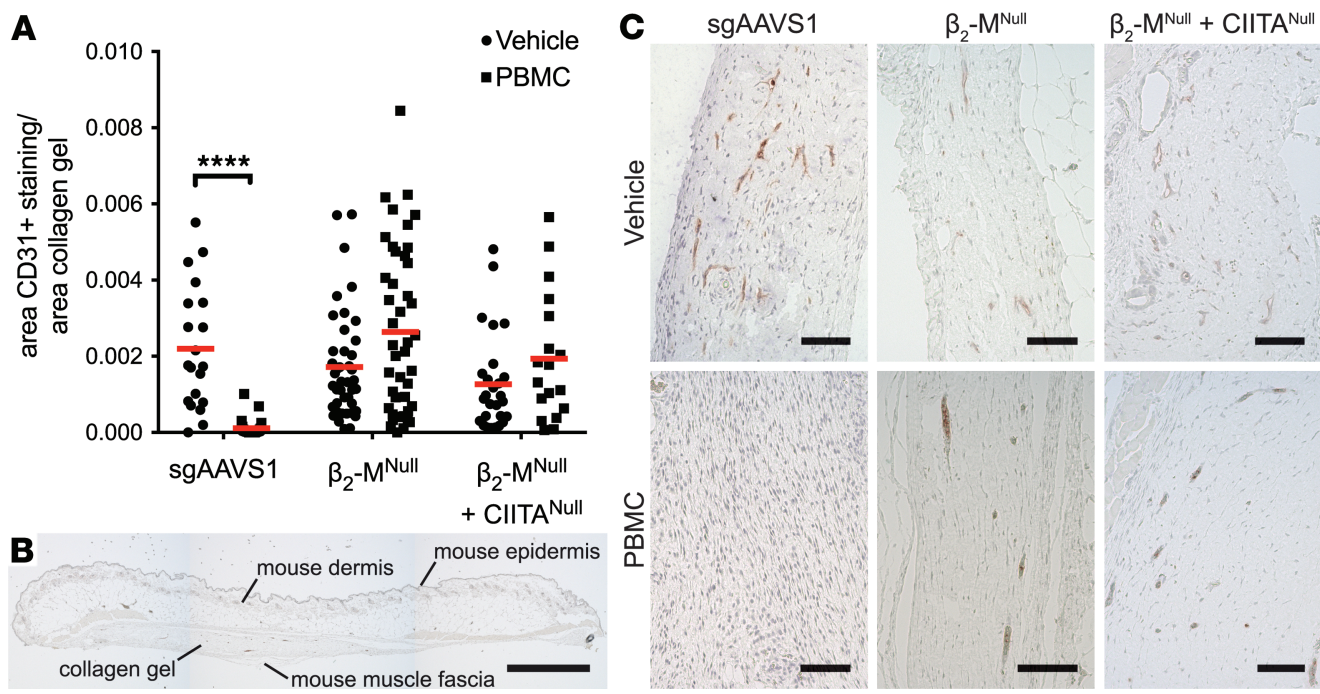

Figure 6. $\beta_{2}-$ Microglobulin ${ }^{\text {null }}$ and $\beta_{2}$-microglobulin ${ }^{\text {null }}+$ CIITA $^{\text {null }}$ ECs evade allogeneic PBMC destruction in a humanized mouse model. (A) Quantification of CD31+ vascular destruction in control, $\beta_{2}$-microglobulin ${ }^{\text {null }}$, and $\beta_{2}$-microglobulin ${ }^{\text {null }}+$ CIITA ${ }^{\text {null }}$ ECs relative to saline-injected mice. Each data point represents the area of CD $31^{+}$vasculature normalized over the area of the collagen gel in a paraffin-embedded $5-\mu \mathrm{m}$ section. A minimum of $30 \mathrm{sec}-$ tions were quantified per collagen gel and at least 3 collagen gels were used per sample. (B) Representative explant of collagen gels containing vasculature from CRISPR/Cas9-modified ECFCs. Scale bar: $100 \mu \mathrm{m}$. (C) Representative images of vascularized collagen gels composed of control (sgAAVS1), $\beta_{2}$-microglobulin ${ }^{\text {null }}$, and $\beta_{2}$-microglobulin ${ }^{\text {null }}+$ CIITA $^{\text {null }}$ ECs in immunodeficient mice injected with vehicle or allogeneic PBMCs. Scale bars: $500 \mu \mathrm{m}$. Three to six animals were used in each group. $\mathrm{CD} 31^{+}$immunohistochemical staining is shown in representative sections demonstrating the presence of vessels in animals treated with vehicle and in those lacking class I MHC, but not those harboring class I MHC in animals engrafted with allogeneic PBMCs.

presence of K562 NK-cell targets (Figure 5D). The mechanism underlying how $\beta_{2}$-microglobulin ${ }^{\text {null }}$ ECs inactivate NK cells while MHC I-sufficient ECs do not is unclear, but this observation has identified an important question for future investigation.

In vivo characterization of allogeneic lymphocyte-mediated responses to CRISPR/Cas9-modified ECs. Recapitulating alloresponses in mouse models is challenging due to the lack of alloreactive memory cells in animals maintained in clean vivaria and the absence of class II MHC molecules on mouse ECs necessary to activate alloreactive $\mathrm{CD} 4^{+} \mathrm{T}$ effector cells $(16,27)$. Assessment of human alloresponses to ECs in vivo can be modeled by embedding human ECs in a collagen/fibronectin protein gel and implanting the neotissue subcutaneously in an immunodeficient C.B-17 SCID/bg mouse, allowing a human microvascular network to establish over a period of 10-14 days, followed by adoptive transfer of PBMCs allogeneic to the ECs (32). The transfer of human PBMCs results in microvascular destruction over a subsequent period of 10 days that is allospecific (33-35). To assess the susceptibility of CRISPR/Cas9-modified ECs to $\mathrm{T}$ cell-mediated responses, control (sgAAVS1), $\beta_{2}$-microglobulin ${ }^{\text {null }}$, or $\beta_{2}$-microglobulin ${ }^{\text {null }}+$ CIITA $^{\text {null }}$ ECs were embedded in a collagen/fibronectin gel and implanted subcutaneously in C.B-17 SCID/bg. Mice implanted with gels were then subjected to saline injection or adoptive transfer of human PBMCs from a donor allogeneic to the ECs. In concordance with in vitro experiments, destruction of synthetic microvessels formed from $\beta_{2}$-microglobulin ${ }^{\text {null }}$ or $\beta_{2}$-microglobulin ${ }^{\text {null }}+$ CIITA $^{\text {null }} E C s$ was not observed, while completed destruction of microvessels was observed in gels containing control ECs (Figure 6).

\section{Discussion}

In this report, we demonstrate that CRISPR/Cas9 targeting of $\beta_{2}$-microglobulin and CIITA in HECFC-derived ECs eliminates class I and II MHC expression and can generate functional ECs that evade alloimmune responses. MHC-absent ECs no longer bind human alloantibody or activate allogeneic $\mathrm{CD} 4^{+} \mathrm{Tem}$ cells, and are resistant to killing by $\mathrm{CD} 8^{+}$alloreactive CTLs in vitro and in vivo. Moreover, the absence of MHC I does not confer susceptibility to allogeneic NK cells. Collectively, these data demonstrate the potential utility of these ECs as cellular components in engineering vascularized grafts. 
The capacity of a graft to perform its vital functions is reliant on its ability to obtain inflow of nutrients and oxygen as well as yield a circulatory outflow to the host. In human tissues, these properties are facilitated by capillary beds lined by ECs that serve as the intermediaries between the vascular space and interstitial tissue. Tissue-engineered epithelialized skin grafts lacking vascularized capillaries can promote healing but characteristically slough due to ischemia. In the absence of ECs, however, they do not sensitize the host to alloantigens expressed on the stromal or parenchymal cells. In contrast, natural human skin is vigorously rejected in a process that targets the EC-lined blood vessels (36). Acute organ graft rejection is largely a cell-mediated process and class I and II MHC molecules expressed on the graft cell luminal surface are the principal proteins recognized by circulating alloreactive Tem cells. Class I molecules are the principal target of CTLs, the primary effectors of cell-mediated rejection. Preformed alloantibodies directed against graft class I and class II MHC molecules, when present, may also contribute to acute rejection. Late graft loss is most commonly attributed to chronic rejection and de novo formation of donor-specific antibodies, usually targeting graft class II MHC molecules, is a major risk factor. The ECs lining graft vessels are the primary cells that express both class I and class II MHC molecules. Potent immunosuppressive therapies are necessary to make clinical organ transplantation feasible. The fundamental premise of this work is that ECs lacking class I and II MHC molecules, even from allogeneic sources, are likely to reduce the potency of rejection, permitting a reduction in the level of immunosuppression required and thus sparing patients from the side effects of these agents.

Tissue engineering will require fashioning of organs that do not provoke strong allogeneic responses. ECs in this process may be differentiated from autologous stem cells or from allogeneic cell sources. Only allogeneic sources will allow off-the-shelf assembly of organs, but technical advances, such as 3D bioprinting, may close the gap. The absence of dendritic cells in such grafts will reduce alloreactivity. However, unlike the experimental situation in rats, where removal of dendritic cells produces immunological ignorance, acute rejection will still occur in humans $(37,38)$. The accepted explanation for this difference is that human graft ECs, unlike rat ECs, can initiate rejection by presentation of non-self MHC molecules to circulating Tem cells. Our data demonstrate that ablation of MHC molecules from human ECs is possible by dual targeting of $\beta_{2}$-microglobulin and CIITA, providing protection from alloimmunity without altering key EC functions.

Interestingly, $\beta_{2}$-microglobulin ${ }^{\text {null }}$ microvessels were completely resistant to destruction by allogeneic PBMCs in our in vivo humanized mouse model of allorejection, despite continued expression of HLA class II molecules and a retained capacity to activate alloreactive $\mathrm{CD} 4^{+} \mathrm{T}$ cells. Coupled with previously published observations that CIITA ${ }^{\text {null }}$ ECs conferred only partial protection from allogeneic PBMCs, these findings suggest that $\mathrm{T}$ cell-mediated cytotoxicity is solely conferred by $\mathrm{CD} 8^{+} \mathrm{T}$ lymphocytes, but whose alloimmune potential is significantly augmented in the presence of $\mathrm{CD} 4^{+} \mathrm{T}$ lymphocytes (19). While other studies have suggested that $\mathrm{CD}^{+} \mathrm{T}$ lymphocytes may directly mediate cytotoxic responses $\left(\mathrm{CD} 4^{+} \mathrm{CTLs}\right)$ in allorejection, they do not appear to function in our model $(39,40)$.

Our study found that dual ablation of $\beta_{2}$-microglobulin and CIITA markedly reduced but did not completely eliminate binding of alloantibody. Serum cytokines such as IL-1 are capable of inducing NF- $\mathrm{kB}$ signaling in the absence of alloantibody and may account for the presence of p100-to-p52 conversion in ECs unexposed to allosensitized serum (26). Moreover, binding of non-HLA antibodies, which can be directed against antigens including MICA/MICB, angiotensin receptor, perlecan, and collagen, as well as mediate antibody-mediated acute and chronic rejection (41), may account for residual alloantibody binding and $\mathrm{NF}-\mathrm{\kappa B}$ signaling noted in evaluation of alloantibody responses to MHC-absent ECs. Whether significant non-HLA responses result de novo or as a consequence of significant ischemia/reperfusion injury, however, is unknown. Whether such responses will limit the ability of MHC-absent ECs to exhibit complete immune ignorance is also unknown and a topic worthy of future investigation.

CRISPR/Cas9 targeting of $\beta_{2}$-microglobulin to eliminate class I MHC molecules has recently been successfully employed in mouse tumor lines for the purposes of generating transplantable tumors across mouse strains (42). As with prior experiments using hematopoietic cell lines, some tumor cells ablated of $\beta_{2}$-microglobulin lose susceptibility to CTL recognition but become prone to NK cell cytotoxicity (28, 43). However, NK responses to vascularized tissues may require activation signals to initiate responses, as has been observed in MHC-deficient tumors (44). Our findings are consistent with observations that solid organ allografts are not rejected in mice by NK cells in the absence of alloreactive T cells that provide activating cytokines or alloantibody that binds to graft ECs even though NK cells can control infections in the same animals $(45,46)$. These experiments contradict the widely expressed view that absent self is a sufficient trigger for NK-mediated rejection, but the original laws of transplantation formulated 
by George Snell distinguished between failure of an F1 offspring of 2 different inbred parents to reject tissue grafts from either parent, with active rejection of hematopoietic cell grafts from the same parent by the same offspring (47). We know that NK cells are responsible for killing the hematopoietic grafts yet fail to reject the tissue graft. We do not know the mechanisms by which ECs avoid activating alloreactive NK cells and our finding that ECs lacking class I MHC may anergize NK cells was unexpected. Wildtype ECs are, as expected, resistant to NK cells but do become susceptible to NK cell-mediated killing when coated with alloantibody. Thus, the lack of MHC molecule expression by $\beta_{2}$-microglobulin ${ }^{\text {null }}$ ECs may actually provide dual protection from both NK cells as well as CTLs. The humanized mouse model we have used to test EC resistance to CTLs in vivo does not have functional human or mouse NK cells. Newer humanized mouse models currently in development may overcome this limitation and enable in vivo testing of more complete human alloimmune responses to composite tissue grafts (48).

Use of ECs, differentiated from cord-blood ECFCs (formerly known as endothelial progenitor cells, a term abandoned because many publications conflated ECFCs with proangiogenic monocytes) has recently garnered interest as a promising tool for vascularization of injured tissue due to their augmented proliferative capacity and ability to form microcapillaries in vivo $(49,50)$. Other investigators have modified induced pluripotent stem (iPS) cells in a similar approach (51). While CRISPR/Cas genetic modification of ECFCs has the potential to introduce off-target mutations, the technology for such engineering is steadily improving with better engineered reagents. While the presence of off-target mutagenesis was not evaluated by whole-genome sequencing of modified cells in this study, we found that modification of MHC-dependent genes in ECFCs produces few unexpected transcriptional changes and does not alter their characteristic functional phenotype. Engineering of committed endothelial progenitors may be safer than modifying iPS cells as an EC source because of the absence of deleterious mutations introduced during the dedifferentiation and redifferentiation process and the absence of a risk for teratoma formation (52).

Utilization of nonimmunogenic ECs could have significant therapeutic potential in 2 major areas in addition to the role for tissue engineering emphasized in our study: (i) as a directed cellular therapy to promote revascularization of an ischemic tissue, and (ii) as a vehicle for efficient delivery of gene products in the circulation. Therapeutic revascularization has the potential to significantly mitigate damage from acute ischemic events including myocardial infarction, stroke, retinal ischemia, and peripheral limb ischemia (53-56). Targeted application of a cellular suspension into affected tissues in rodent animal models have demonstrated improvement in organ function and increased vascularization of affected tissues, particularly when coimplanted with host myeloid cells or smooth muscle cells $(57,58)$. In addition to aiding in vasculogenesis, secretion of soluble regulators governing tissue repair may present additional benefits. For example, engraftment of pancreatic islets under the renal capsule of NOD/SCID mice in the presence of ECFC-derived ECs promoted $\beta$ cell proliferation and improved glycemic control and C-peptide production in these animals (59). These effects are likely due to paracrine provision of signals by the ECs. Recent studies have also evaluated the utility of ECs as vehicles for exogenous gene delivery. Transfected ECFCs carrying modified coagulation factor VIII were able to engraft in NOD/SCID mice and efficiently secrete factor VIII into the circulation to achieve therapeutic levels relevant for treatment of hemophilia within 5 months (60). Genetically engineered ECFCs optimized for delivery of erythropoietin and embedded in collagen/ fibrin gels in immunodeficient mice have shown the ability to augment hematocrit, red blood cell counts, and splenic extramedullary erythropoiesis for treatment of nephrectomy-induced anemia (61).

In summary, we have shown that the alloimmunogenic properties of ECs may be eliminated by ablation of MHC molecules, enhancing their utility for vascularization of human tissue engineered constructs as well as for promoting tissue regeneration or therapeutic protein delivery (62).

\section{Methods}

Culture of human ECs derived from cord-blood ECFCs. Late-outgrowth ECFCs were collected from cord blood and cultured as described previously (63). Colonies of proliferating, differentiated cells were typically identified at 10 days, at which time the medium was changed to EGM2MV Complete Medium (Lonza) for expansion and propagation.

CRISPR/Cas9-mediated ablation of $\beta_{2}$-microglobulin and CIITA. Guide RNA sequences in the exonic regions of the $\beta_{2}$-microglobulin and CIITA gene loci were identified using the design software available at crispr.mit. edu. Sequences with perfect matches in the human genome and at least 3 mismatches to the nearest coding off-target sequences were selected and used to create gBlocks segments (IDT) containing an XhoI restriction site, 
U6 promoter, sgRNA target sequences, and an NheI restriction site in consecutive order from the $5^{\prime}$ to $3^{\prime}$ end. gBlocks were subcloned into pLX-sgRNA (produced by Eric Lander and David Sabatini and available through Addgene as plasmid 50662), which contains a blasticidin resistance gene. Guide strand targeting sequences included GAGTAGCGCGAGCACAGCTA for targeting of the $\beta_{2}$-microglobulin gene locus, GATATTGGCATAAGCCTCCC for targeting of the CIITA gene locus, and GGGGCCACTAGGGACAGGAT for targeting of the AAVS1 gene locus (used as a control). A separate lentiviral vector encoding tetracycline-inducible FLAG-Cas9 and a puromycin resistance gene (pCW-Cas9, produced by Eric Lander and David Sabatini and available through Addgene as plasmid 50661) was used to create TetOn-Cas9-ECs.

pCW-Cas9 or (AAVS1-, $\beta_{2}$-microglobulin-, or CIITA-targeting) pLX-sgRNA lentiviral vector plasmids were cotransfected with psPAX2 (produced by Didier Trono, available as Addgene plasmid 12260) and CMV VSV-G-containing plasmid pMD2.G (produced by Didier Trono, available as Addgene plasmid 12259) packaging and envelope plasmids into human 293T (ATCC) cells in a 1:1 mixture of plasmids and a calcium phosphate-based transfection buffer ( $4 \mathrm{mg} \mathrm{NaCl}, 190 \mu \mathrm{g} \mathrm{KCl}, 50 \mu \mathrm{g} \mathrm{Na} \mathrm{HPO}_{4}, 2.5 \mathrm{mg} \mathrm{HEPES}$, $500 \mu$ g glucose, $125 \mu \mathrm{mol} \mathrm{CaCl}$ in $500 \mu \mathrm{L}, \mathrm{pH}$ 7.05). Lentiviral supernatant was collected at 48 and 72 hours and filtered through a $0.45-\mu \mathrm{m}$ filter. Polybrene (Sigma-Aldrich) was added to a final concentration of $8 \mu \mathrm{g} / \mathrm{mL}$. pCW-Cas9 and AAVS1-, $\beta_{2}$-microglobulin-, or CIITA-targeting pLX-sgRNA supernatant was simultaneously cotransduced into ECs for an 8-hour incubation at MOIs of 30 and 20, respectively (Lenti-X p24 Lentiviral Titration Kit, Clontech). Following a 16-hour recovery period, a second 8-hour transduction was carried out, after which transduced ECs were selected with $1 \mu \mathrm{g} / \mathrm{mL}$ puromycin (Invitrogen) for 72 hours. AAVS1, $\beta_{2}$-microglobulin, CIITA, or $\beta_{2}$-microglobulin and CIITA Cas9-ECs subsequently underwent a second selection with $10 \mu \mathrm{g} / \mathrm{mL}$ blasticidin (Sigma-Aldrich) for 5 days, performed concurrently with induction of TetOn-Cas9 with $1 \mu \mathrm{g} / \mathrm{mL}$ doxycycline (Sigma-Aldrich) for 10 days.

Flow cytometry and isolation of CRISPR/Cas9-modified ECs. After antibiotic selection and Cas9 induction with doxycycline, ECs were cultured in the presence of $50 \mathrm{ng} / \mathrm{mL}$ IFN- $\gamma$ (Gibco) and collected after treatment with trypsin (TrypLE Express, Invitrogen). Cells were costained with directly conjugated eFluor450 Viability dye (Invitrogen), FITC-anti-HLA-A,B,C (clone W6/32, BioLegend), and Alexa Fluor 647-antiHLA-DR (clone L243, BioLegend). $\beta_{2}$-Microglobulin and CIITA loss of function was identified by the presence of live cells that increased expression of HLA-DR and HLA-A, -B, -C (in the case of the AAVS1 control vector), increased expression of HLA-DR and not HLA-A, -B, -C (in the case of $\beta_{2}$-microglobulin), or increased expression of HLA-A, -B, -C and not HLA-DR (in the case of CIITA), or no expression of either (in the case of $\beta_{2}$-microglobulin and CIITA dually targeted cells), with the positive threshold defined by fluorescence-minus-one staining (Supplemental Figure 1). These gates were used to collect $\beta_{2}$-microglobulin $^{+/+}$CIITA $^{+/+}, \beta_{2}$-microglobulin ${ }^{\text {null }}$, CIITA $^{\text {null }}$, and $\beta_{2}$-microglobulin ${ }^{\text {null }}+$ CIITA $^{\text {null }}$ ECs using a low-pressure $100-\mu \mathrm{m}$ nozzle by FACS (BD Biosciences, FACSAria II). Single cells were deposited into each well of a C96-well flat-bottom plate containing EGM2MV Complete Medium supplemented with $5 \mu \mathrm{M}$ ROCK-selective inhibitor Y-27632 (Sigma-Aldrich). EGM2MV Complete Medium was then exchanged every 48 hours and colonies were serially expanded into larger vessels over the course of 2 to 3 weeks.

For functional assays and immune assays that did not involve RNA sequencing or deep sequencing, CRISPR/Cas9-transduced cells that did not exhibit biallelic loss of function were isolated by antibody depletion. Unsorted, pooled $\beta_{2}$-microglobulin ${ }^{+/+} \mathrm{CIITA}^{+/+}, \beta_{2}$-microglobulin ${ }^{\text {null }}$, CIITA $^{\text {null }}$, or $\beta_{2}$-microglobu$\operatorname{lin}^{\text {null }}+$ CIITA ${ }^{\text {null }}$ ECs were treated for 72 hours with $50 \mathrm{ng} / \mathrm{mL} \mathrm{IFN}-\gamma$ and incubated with $10 \mu \mathrm{g} / \mathrm{mL}$ control antibody (MOPC-173, BioLegend), anti-HLA-A,B,C (clone W6/32, BioLegend), anti-HLA-DR (clone L243, BioLegend), or both anti-HLA-A,-B,-C and anti-HLA-DR, respectively. Positive cells were depleted using pan-mouse IgG Dynabeads (Invitrogen), according to the manufacturer's protocol.

PCR and deep CRISPR/Cas9 sequencing. Genomic DNA was isolated from clonally expanded ECs in a C96-well plate using $200 \mu \mathrm{L}$ QuickExtract DNA Extraction Solution (Epicentre). A DNA fragment flanking both sides of the target sequence and Cas9 protospacer-adjacent motif was amplified by PCR (Platinum PCR SuperMix High Fidelity, Invitrogen) according to the manufacturer's protocol. Forward and reverse primer pairs used to evaluate a 280-bp fragment of the $\beta_{2}$-microglobulin gene locus were CTGGCTTGGAGACAGGTGAC and GAAGTCACGGAGCGAGAGAG, respectively. Forward and reverse primer pairs used to evaluate a 180-bp fragment of the CIITA gene locus were AGACACCATCAACTGCGACC and CGTGGCTCATGATGAATGGG, respectively. PCR amplicons were purified using a QIAquick PCR Purification Kit (Qiagen) and submitted for deep CRISPR sequencing at the MGH CCIB DNA Core (Cambridge, Massachusetts, USA). 
$R N A$ sequencing. Sorted and clonally expanded CRISPR/Cas9-modified EC colonies were grown to confluence on a C12-well plate and treated with $50 \mathrm{ng} / \mathrm{mL}$ IFN- $\gamma$ for 96 hours. RNA was extracted and purified using a RNeasy Mini kit (Qiagen), in which an on-column DNase treatment was included. Six clonally derived colonies, derived from $2 \mathrm{EC}$ donors, were included for each of the following conditions: $\beta_{2}$-microglobulin ${ }^{+/+} \mathrm{CI}-$ ITA $^{+/+}, \beta_{2}$-microglobulin ${ }^{\text {null }}$, CIITA $^{\text {null }}$, and $\beta_{2}$-microglobulin ${ }^{\text {null }}+$ CIITA $^{\text {null }}$. Twenty-four strand-specific sequencing cDNA libraries (6 per condition) were produced from purified total RNA samples by the Illumina TruSeq stranded protocol. The libraries underwent 101-bp pair-end sequencing using an Illumina HiSeq 4000 according to Illumina's protocols, generating an average of 33 million paired-end reads per library. For each read, we trimmed the first 6 nucleotides and the last nucleotides at the point where the Phred score of an examined base fell below 20 using in-house scripts. If after trimming the read was shorter than $45 \mathrm{bp}$, the read was discarded. Trimmed reads were mapped to the human reference genome (hg19) with a known transcriptome index (UCSC Known Gene annotation) with Tophat v2.1.1 (64) using the very-sensitive preset, first-strand library type, and providing the corresponding gene model annotation. Only reads that mapped to a single unique location within the genome, with a maximum of 2 mismatches in the anchor region of the spliced alignment, were reported in these results. We used the default settings for all other Tophat options. Tophat alignments were then processed by Cuffdiff (Cufflinks v2.2.1; ref. 65) to obtain differential gene expression using first-strand library type, providing gene model annotation and the genome sequence file for detection and correction of sequence-specific bias that random hexamers can cause during library preparation. $P$ values were adjusted for multiple hypothesis testing using the Benjamini-Hochberg procedure (66). Sequencing and analysis results were deposited in NCBI's Gene Expression Omnibus (GEO) under accession number GSE130443.

Immunofluorescence microscopy. ECs were grown to confluence on gelatin-coated glass coverslips in C24well plates and fixed in $95 \%$ ethanol for 30 minutes. Fixed monolayers were incubated overnight in $5 \mu \mathrm{g} /$ $\mathrm{mL}$ mouse anti-human CD31 (clone WM59, BioLegend) and CD144 (clone BV9, BioLegend) diluted in Tris-buffered solution/0.2\% Triton X-100/5\% normal donkey serum. Donkey Alexa Fluor 488 and Alexa Fluor 594 anti-mouse secondary antibodies were used to detect the primary antibody. Coverslips were mounted for analysis in ProLong mounting medium (Invitrogen) and randomly selected fields were collected by a Zeiss Axiovert fluorescence microscope with an ORCA-ER digital camera (Hamamatsu Photonics).

Electrical cell-substrate impedance sensing assays. ECs were grown to confluence on 96-well plates (8W20idf PET, Applied BioPhysics). Transendothelial electrical resistance of ECFCs was assessed by a 96-well electrical cell-substrate impedance sensor (model Z- $\theta$, Applied BioPhysics). Confluent cell monolayers were stimulated with $10 \mathrm{ng} / \mathrm{mL}$ TNF- $\alpha$ (Invitrogen) or $1 \mathrm{U} / \mathrm{L}$ thrombin (Invitrogen). Data were acquired using a Z- $\theta$ instrument with ECIS software (Applied BioPhysics).

Flow cytometry analysis and Western blot characterization of ECS. Colonies expanded from single-cell clones were further evaluated for the presence of class I and II MHC molecules by surface and intracellular staining for class I heavy chain (clone EMR8-5, Abcam) and class II heavy chain (clone XD5.A11 ascites, gifted by Peter Cresswell, Yale University) following fixation and permeabilization (Intracellular Fixation and Permeabilization Kit, BioLegend). ECs were also characterized for expression of HLA-DQ (BioLegend, clone Tu169) and HLA-DP (Abcam, clone B7/21) by flow cytometry, according to the manufacturers' protocols. $\beta_{2}$-Microglobulin ${ }^{+/+}$CIITA $^{+/+}, \beta_{2}$-microglobulin ${ }^{\text {null }}$, CIITA $^{\text {null }}$, or $\beta_{2}$-microglobulin ${ }^{\text {null }}+$ CIITA $^{\text {null }}$ ECs were also costained with Pacific Blue-conjugated anti-CD54 (BioLegend, clone HA58) and APC-conjugated anti-CD274 (BioLegend, clone 29E.2A3). Stained cells were evaluated in triplicate using 4 independent donors with a minimum of $1 \times 10^{5}$ live cells per sample acquired on an LSR II (BD Biosciences). All assays were carried out following 72 hours of treatment with $50 \mathrm{ng} / \mathrm{mL}$ IFN- $\gamma$.

To determine whether $\beta_{2}$-microglobulin ${ }^{\text {null }}$ ECs were able to reconstitute class I MHC in trans, cells were cultured for 72 hours in the presence of EGM2MV media (Lonza) supplemented with 15\% human ABO serum (Sigma-Aldrich) and $5 \mu \mathrm{g} / \mathrm{mL}$ recombinant human $\beta_{2}$-microglobulin (Abcam). ECs were characterized for expression of HLA-A, -B, -C (clone W6/32, BioLegend) by flow cytometry as described above.

The presence of an unfolded protein response was examined by Western blot by collecting $1 \times 10^{5} \mathrm{ECs}$ treated with vehicle, $50 \mathrm{ng} / \mathrm{mL}$ IFN- $\gamma$ for 72 hours, or $2 \mu \mathrm{M}$ thapsigargin (Sigma-Aldrich) for 6 hours were lysed in $50 \mu \mathrm{L}$ of Laemmli buffer (Bio-Rad). Ten microliters were run in a $12 \%$ SDS gel and transferred onto a PVDF membrane overnight. Membranes were blocked with 5\% BSA in TBS and incubated with $1 \mu \mathrm{g} / \mathrm{mL}$ anti-human CHOP antibody (Cell Signaling Technology, clone L63F7) overnight at $4^{\circ} \mathrm{C}$ and $100 \mathrm{ng} / \mathrm{mL}$ horseradish peroxidase-conjugated anti-mouse secondary antibody (Jackson Laboratories) at room temperature for 2 hours and visualized using Femto Western Maximum Sensitivity Substrate (Thermo Fisher Scientific). 
Alloantibody assays. ECs were stimulated for 72 hours with $50 \mathrm{ng} / \mathrm{mL}$ IFN- $\gamma$ and plated to confluence in a $\mathrm{C} 12$ well. In experiments evaluating alloantibody binding to ECs, cells were treated with either $25 \%$ unsensitized human ABO serum (Sigma-Aldrich) or 25\% high-titer PRA serum (calculated PRA [cPRA] > $80 \%$ ) isolated and pooled from multiple allosensitized transplant candidates diluted in gelatin veronal buffer. In compliance with Health Insurance Portability and Accountability Act (HIPPA) regulations, cPRA > $80 \%$ sera, as determined by Luminex single antigen testing (One Lambda), were collected and pooled from multiple individuals by the Yale-New Haven Hospital Histocompatibility Laboratory. Following a 2-hour incubation at $37^{\circ} \mathrm{C}$, cells were harvested and stained with $5 \mu \mathrm{g} / \mathrm{mL}$ FITC-anti-human IgG (Abcam) or mouse anti-human C4d (clone A213, Quidel). Cells stained with the latter antibody were then incubated with $5 \mu \mathrm{g} / \mathrm{mL}$ Alexa Fluor 647-anti-mouse antibody (Life Technologies). Samples were analyzed on an LSR II flow cytometer (BD Biosciences). To evaluate noncanonical NF- $\mathrm{kB}$ signaling, $1 \times 10^{5}$ Cas9-modified cells treated with gelatin veronal buffer or $25 \%$ high-titer cPRA serum were lysed in $100 \mu \mathrm{L}$ of Laemmli buffer. Fifteen microliters was run in a 5\%-20\% SDS gel and transferred onto a PVDF membrane overnight. Membranes were blocked with 5\% BSA in TBS and incubated with $1 \mu \mathrm{g} / \mathrm{mL}$ anti-human p100/ p52 antibody (Cell Signaling Technology, clone 18D10) overnight at $4^{\circ} \mathrm{C}$ and $100 \mathrm{ng} / \mathrm{mL}$ horseradish peroxidase-conjugated anti-rabbit secondary antibody at room temperature for 1 hour and visualized using Femto Western Substrate. Densitometry was quantified from scanned images using ImageJ (NIH).

Isolation of human $\mathrm{CD}^{+}$and $\mathrm{CD} 8^{+}$Tem cells. PBMCs were collected by leukapheresis from anonymized healthy human volunteers and enriched by density centrifugation using Lymphocyte Separation Medium (MP Biomedicals) according to the manufacturer's protocol, and subsequently cryopreserved in liquid nitrogen after resuspension in 90\% FBS (VWR)/10\% DMSO (J.T. Baker).

PBMCs were thawed and $\mathrm{CD}^{+}$and $\mathrm{CD}^{+} \mathrm{T}$ lymphocytes were isolated using a Dynabeads CD4 or CD8 Positive Isolation Kit (Invitrogen). Removal of naive $\mathrm{T}$ cells, central memory $\mathrm{T}$ cells, and activated and $\mathrm{CD}^{+} \mathrm{CD}^{+}$lymphocytes was achieved by negative selection following incubation with mouse antiCD45RA (clone H100, $5 \mu \mathrm{g} / \mathrm{mL}$ ), anti-CCR7 (clone G04387, $10 \mu \mathrm{g} / \mathrm{mL}$ ), anti-CD62L (clone DREG-56, $5 \mu \mathrm{g} / \mathrm{mL}$ ), anti-HLA-DR (clone L243, $5 \mu \mathrm{g} / \mathrm{mL}$ ), anti-CD25 (clone BC96, $5 \mu \mathrm{g} / \mathrm{mL}$ ), and either anti-CD4 (clone SK3, $5 \mu \mathrm{g} / \mathrm{mL}$ ) or anti-CD8 (clone SK1, $5 \mu \mathrm{g} / \mathrm{mL}$ ) (all from BioLegend) and subsequent depletion using pan-mouse IgG beads (Invitrogen). Isolated Tem cells were routinely greater than $95 \% \mathrm{CD}^{2} 5 \mathrm{RO}^{+} \mathrm{C}$ CR7-CD62L-HLA-DR ${ }^{-} \mathrm{CD} 25^{-} \mathrm{CD}^{+}{ }^{+}$or $\mathrm{CD}^{-}$25O ${ }^{+} \mathrm{CCR} 7^{-} \mathrm{CD} 62 \mathrm{~L}^{-} \mathrm{HLA}-\mathrm{DR}{ }^{-} \mathrm{CD} 25^{-} \mathrm{CD} 4^{+} \mathrm{CD} 8^{+}$(Supplemental Figure 3). In lymphocyte assays, Tem cells were cultured with ECs in RPMI 1640 medium (Gibco) with 10\% FBS, 2\% L-glutamine (Sigma-Aldrich), and 1\% penicillin-streptomycin (Invitrogen).

Mixed EC-lymphocyte proliferation and activation assays. ECs were plated at confluence $\left(1 \times 10^{5}\right.$ cells/well $)$ into C24-well tissue culture plates and stimulated with $50 \mathrm{ng} / \mathrm{mL}$ IFN- $\gamma$ for 72 hours. Unfractionated PBMCs or purified $\mathrm{CD}^{+}$or $\mathrm{CD} 8^{+}$Tem cells were labeled at $37^{\circ} \mathrm{C}$ using the Cell Trace Violet Cell Proliferation Kit (Invitrogen). Labeled CellTrace Violet ${ }^{+}$effector cells were then added to wells containing EC monolayers at an effector/target ratio of 30. Coculture was maintained for 7 days in $1 \mathrm{~mL}$ of RPMI 1640 medium with $10 \%$ FBS, $2 \%$ L-glutamine, and 1\% penicillin-streptomycin. Lymphocytes were subsequently costained with an eFluor780 viability dye (eBioscience), APC anti-CD4 (clone SK3, BioLegend), anti-CD8 (clone SK1, BioLegend), anti-HLA-DR (clone L243, BioLegend), and analyzed by flow cytometry (LSR II).

Generation of CTLs and cytotoxicity assays. CD8 ${ }^{+}$lymphocytes were isolated from PBMCs using a Dynabeads CD8 Positive Isolation Kit and cocultured with allogeneic ECs in C24-well plates at a responder/stimulator ratio of 20 in RPMI 1640 medium with 10\% FBS, 2\% L-glutamine, and 1\% penicillin-streptomycin. The medium was supplemented with IL-2 (Gibco) to a final concentration to $6 \mathrm{U} / \mathrm{mL}$ on day 3. On day 7 , the medium was changed and CTLs were transferred to fresh stimulator cells from the same stimulating donor. The medium was supplemented with IL-2 to a final concentration of $6 \mathrm{U} / \mathrm{mL}$ on day 10 . T lymphocytes were re-isolated sing a Dynabeads CD8 Positive Isolation Kit on day 14 and used for cytotoxicity assays.

Approximately $2 \times 10^{4}$ ECs were stimulated for 72 hours with $50 \mathrm{ng} / \mathrm{mL}$ IFN- $\gamma$ in a C96-well U-bottom microtiter plate (Corning). Target ECs were labeled with $2 \mu \mathrm{M}$ calcein AM for 30 minutes (Invitrogen) and differentiated CTLs raised on donor-matched ECs were added in $100 \mu \mathrm{L}$ RPMI medium (without phenol red) supplemented with $5 \mathrm{mM}$ HEPES at 10:1, 3:1, 1:1, and 0.3:1 effector/target ratios. Following 4 hours of coculture, $75 \mu \mathrm{L}$ of supernatant was collected and read on a fluorescent-plate reader. Eight replicates were used for each condition. The percentage of cytotoxicity was defined as (dead - experimental)/ (dead-live), where live corresponds to the value of calcein-loaded ECs without CTL, experimental is with CTL, and dead denotes ECs lysed with 1\% Triton X-100 (Sigma-Aldrich). 
Isolation of human NK cells, ELISAs, and cytotoxicity analysis. NK cells were isolated from thawed PBMCs using an EasySep Human NK Cell Enrichment Kit (Stemcell Technologies) according to the manufacturer's protocol. The NK-enriched cell population was further depleted of remaining $\mathrm{CD}^{+}$lymphocytes with CD3 Dynabeads (Invitrogen). Isolated NK cells were routinely greater than $90 \% \mathrm{CD}^{-} \mathrm{CD}^{-} 6^{+}$and expressed low levels of CD335 (Supplemental Figure 3).

ECs were stimulated for 72 hours with $50 \mathrm{ng} / \mathrm{mL}$ IFN- $\gamma$ in a C24-well microtiter plate (Corning). Purified, unactivated, human NK cells were then cocultured with each respective EC cell type or K562 lymphoblasts (ATCC) in RPMI 1640 medium with 10\% FBS, 2\% L-glutamine, and 1\% penicillin-streptomycin at an effector/target ratio of 20 for 6 hours in a volume of $0.2 \mathrm{~mL}$. Supernatants were collected and run in duplicate using human TNF- $\alpha$ or IFN- $\gamma$ ELISA kits (Invitrogen) according to the manufacturer's protocol.

For cytotoxicity assays, confluent monolayers of ECs were stimulated for 72 hours with $50 \mathrm{ng} / \mathrm{mL}$ IFN- $\gamma$ in a C96-well plate (Corning). A set of $\beta_{2}$-microglobulin ${ }^{+/+} \mathrm{CIITA}^{+/+} \mathrm{ECs}$ were incubated for 15 minutes with $25 \%$ high-PRA serum to serve as a positive control. All ECs were labeled with $2 \mu \mathrm{M}$ calcein AM for 30 minutes and cocultured with purified NK cells for 6 hours at an effector/target ratio of 20. Release of fluorescent dye was measured using a plate reader (BioTek) in bottom-reading mode. The percentage of cytotoxicity was defined as (live - experimental)/(live - lysed) $\times 100$, where live is the value of calcein-loaded ECs without CTLs, experimental is with CTLs, and lysed is the number of lysed cells treated with $1 \%$ Triton X-100.

Wild-type ECFCs and adult HDMECs were compared after 48 hours of treatment with $10 \mathrm{ng} / \mathrm{mL}$ TNF- $\alpha$ (R\&D Systems), $50 \mathrm{ng} / \mathrm{mL}$ IFN- $\gamma$, or PBS with resting K562 for expression of NK ligands by staining with APC-HLA-E (clone 3D12), APC-HLA-G (clone 87G) APC-MICA/MICB (clone 6D4), PE-CD111 (clone R1.302), APC-CD112 (clone TX31), APC-CD155 (clone SKII.4), or with an appropriately conjugated isotype control (all from BioLegend), according to the manufacturer's instructions. The effects of TNF- $\alpha$ or IFN- $\gamma$ were confirmed by staining for PE-VCAM-1/CD106 (clone STA) and APCPD-L1/CD274 (clone B7-H1), respectively, antigens known to be upregulated in ECs. Human dermal microvascular cells were isolated as previously described (67).

In an assay performed to distinguish immune inhibition from induction of anergy of NK cells, isolated NK cells were cultured on confluent monolayers of $\beta_{2}$-microglobulin ${ }^{+/+} \mathrm{CITA}^{+/+}, \beta_{2}$-microglobulin ${ }^{\text {null }}$, or no-target cells at a concentration of $1 \times 10^{6}$ cells $/ \mathrm{mL}$ for 6 hours in RPMI/10\% FBS. NK cells were then harvested and cocultured with $2 \times 10^{5} \mathrm{~K} 562$ cells per C48 well at an effector/target ratio of 5 or a well lacking target cells in a total volume of $0.2 \mathrm{~mL}$. Supernatant was harvested at 6 hours and run in duplicate using a human IFN- $\gamma$ ELISA kit (Invitrogen) according to the manufacturer's protocol. This was performed in 2 independent donor pairs.

Synthetic microvessel formation and in vivo implantation. Human microvessels composed of $\beta_{2}$-microglobulin $^{+/+}$CIITA $^{+/+}, \beta_{2}$-microglobulin ${ }^{\text {null }}$, CIITA $^{\text {null }}$, or $\beta_{2}$-microglobulin ${ }^{\text {null }}+$ CITTA $^{\text {null }}$ ECs were embedded in a collagen/fibronectin gel and implanted subcutaneously in the abdominal wall of 6- to 8-weekold female C.B-17 SCID/bg mice, as previously described (32). In brief, CRISPR/Cas9-modified ECs were suspended in rat tail type I collagen (Corning), human plasma fibronectin (EMD Millipore), and M199 medium (Gibco) in a $0.5 \mathrm{~mL}$ cellular suspension and polymerized at $37^{\circ} \mathrm{C}$ for 30 minutes prior to implantation. In one experiment, each mouse received a single implant of $\beta_{2}$-microglobulin ${ }^{+/+} \mathrm{CIITA}^{+/+}$, $\beta_{2}$-microglobulin ${ }^{\text {null }}$, CIITA $^{\text {null }}$, or $\beta_{2}$-microglobulin ${ }^{\text {null }}+$ CIITA $^{\text {null }}$ ECs. In a separate experiment, each mouse received 2 gel implants to compare $\beta_{2}$-microglobulin ${ }^{+/+}$CIITA $^{+/+}$and $\beta_{2}$-microglobulin ${ }^{\text {null }}+$ CIITA $^{\text {null }}$ ECs. Fourteen days after implantation, one gel was harvested from each mouse to confirm vessel formation 10 minutes after intravenous inoculation with fluorescein-UEA (Vector Laboratories), which labels perfused human vessels. After confirmation of vessel formation, implanted mice were inoculated with PBS or 120 million PBMCs. Animals were euthanized approximately 10 days after inoculation and EC-containing protein gels were fixed in 10\% neutral buffered formalin (Sigma-Aldrich) and embedded in paraffin. Sections of 5 - $\mu \mathrm{m}$ thickness were cut and immunoperoxidase staining for human CD31 (clone JC/70A, Dako) was performed with a hematoxylin counterstain (Vector Laboratories). Tiled microscope images of stained tissue sections were taken in bright-field mode using an EVOS FL Auto2 imaging system (Thermo Fisher Scientific). Forty to sixty randomly selected sections sampling all parts of the tissue block were evaluated per animal. Whole sections were imaged using a $\times 40$ objective (Olympus UPlanSApo, $0.95 \mathrm{NA}$ ), with an image pixel size of $0.175 \mu \mathrm{m}$. A MATLAB code was employed to quantify the vessel-to-gel area ratio in each section. Briefly, the collagen gel region was first defined in the raw image using the MATLAB function roipoly and gel area was calculated by multiplying with the squared pixel size. The green image channel was subtracted from the red channel, resulting in 
an image with the highest intensity for stained vessels. The gel region was then binarized by defining a threshold and holes in adjacent regions were filled. The vessel area was defined as the number of nonzero pixels in the resulting image multiplied by the squared pixel size. The vessel number was quantified by the number of $\mathrm{CD} 31^{+}$vessels normalized to the total gel area. Three to six animals per group were evaluated to observe statistical differences.

Statistics. Data are expressed as mean \pm 1 SEM. Continuous variables were compared using a 2-tailed Student's $t$ test or ANOVA, as appropriate. Analyses were conducted with GraphPad Prism v7.0 and statistical significance was claimed when $P<0.05$.

Study approval. Protocols involving collection and experimentation with human cells were approved by the Yale Human Investigation Committee. All animal protocols were approved by the Yale Institutional Animal Care and Use Committee.

\section{Author contributions}

JSP and JM conceived the study and wrote the manuscript. JM, WMS, GTT, GT, and JSP aided in the experimental design. JM, RWP, LQ, TB, TDM, GL, LGB, and NKS conducted the experiments. JM, MR, SS, FLG, and CX performed critical analyses. JM and JSP wrote the manuscript and all other authors provided critical revisions.

\section{Acknowledgments}

This work is supported by grants from the NIH: R01-HL085416 (to JSP and WMS) and UL1-TR000142 and T32-DK007276 (to JM). This work was also supported by the HPC facilities operated by the Yale Center for Research Computing and the Yale Center for Genome Analysis, as well as NIH grant 1S10OD018521-01, which helped fund the cluster. We thank Gwendolyn Davis-Arrington for assistance with endothelial cell isolation. We acknowledge Zuzanna Tobiasova and Ewa Menet for help with FACS sorting as well as Peter Cresswell for providing anti-human MHC class II heavy chain antibodies.

Address correspondence to: Jordan S. Pober, Department of Immunobiology, Yale School of Medicine, 10 Amistad Street, Room 401D, New Haven, Connecticut 06520-8089, USA. Phone: 203.737.2292; Email: jordan.pober@yale.edu.

1. Suthanthiran M, Strom TB. Renal transplantation. N Engl J Med. 1994;331(6):365-376.

2. Dienstag JL, Cosimi AB. Liver transplantation--a vision realized. N Engl J Med. 2012;367(16):1483-1485.

3. Sawyer J. Heart transplantation. N Engl J Med. 1987;316(23):1480-1481.

4. Stephan A. Organ shortage: Can we decrease the demand? Exp Clin Transplant. 2017;15(Suppl 1):6-9.

5. [No authors listed]. OPTN/SRTR 2016 Annual Data Report. US Department of Health and Human Services. https://srtr. transplant.hrsa.gov/annual_reports/2016_ADR_Preview.aspx. Accessed September 19, 2019.

6. Asahara T, et al. Isolation of putative progenitor endothelial cells for angiogenesis. Science. 1997;275(5302):964-967.

7. Prasain N, et al. Differentiation of human pluripotent stem cells to cells similar to cord-blood endothelial colony-forming cells Nat Biotechnol. 2014;32(11):1151-1157.

8. Banno K, Yoder MC. Tissue regeneration using endothelial colony-forming cells: promising cells for vascular repair. Pediatr Res 2018;83(1-2):283-290.

9. Giwa S, et al. The promise of organ and tissue preservation to transform medicine. Nat Biotechnol. 2017;35(6):530-542.

10. Akalin E. A new treatment option for highly sensitized patients awaiting kidney transplantation. Am J Kidney Dis. 2018;71(4):458-460

11. Keith DS, Vranic GM. Approach to the highly sensitized kidney transplant candidate. Clin J Am Soc Nephrol. 2016;11(4):684-693.

12. Racusen LC, Regele H. The pathology of chronic allograft dysfunction. Kidney Int Suppl. 2010;(119):S27-S32.

13. Jane-Wit D, et al. Alloantibody and complement promote T cell-mediated cardiac allograft vasculopathy through noncanonical nuclear factor- $\kappa \mathrm{B}$ signaling in endothelial cells. Circulation. 2013;128(23):2504-2516.

14. Gaston RS, et al. Evidence for antibody-mediated injury as a major determinant of late kidney allograft failure. Transplantation. 2010;90(1):68-74.

15. Poggio ED, et al. Pretransplant cellular alloimmunity as assessed by a panel of reactive T cells assay correlates with acute renal graft rejection. Transplantation. 2007;83(7):847-852.

16. Shiao SL, Kirkiles-Smith NC, Shepherd BR, McNiff JM, Carr EJ, Pober JS. Human effector memory CD4 ${ }^{+}$T cells directly recognize allogeneic endothelial cells in vitro and in vivo. J Immunol. 2007;179(7):4397-4404.

17. Dengler TJ, Pober JS. Human vascular endothelial cells stimulate memory but not naive CD8 ${ }^{+} \mathrm{T}$ cells to differentiate into CTL retaining an early activation phenotype. J Immunol. 2000;164(10):5146-5155.

18. Biedermann BC, Pober JS. Human vascular endothelial cells favor clonal expansion of unusual alloreactive CTL. J Immunol. 1999;162(12):7022-7030.

19. Abrahimi P, et al. Blocking MHC class II on human endothelium mitigates acute rejection. JCI Insight. 2016;1(1):e85293. 
20. Pober JS, Tellides G. Participation of blood vessel cells in human adaptive immune responses. Trends Immunol. 2012;33(1):49-57.

21. Abrahimi P, et al. Efficient gene disruption in cultured primary human endothelial cells by CRISPR/Cas9. Circ Res. 2015;117(2):121-128.

22. Li X, Faustman D. Use of donor beta 2-microglobulin-deficient transgenic mouse liver cells for isografts, allografts, and xenografts. Transplantation. 1993;55(4):940-946.

23. Anderson DA, Grajales-Reyes GE, Satpathy AT, Vasquez Hueichucura CE, Murphy TL, Murphy KM. Revisiting the specificity of the MHC class II transactivator CIITA in classical murine dendritic cells in vivo. Eur J Immunol. 2017;47(8):1317-1323.

24. Kilgore KS, et al. Sublytic concentrations of the membrane attack complex of complement induce endothelial interleukin-8 and monocyte chemoattractant protein-1 through nuclear factor-kappa B activation. Am J Pathol. 1997;150(6):2019-2031.

25. Kilgore KS, Flory CM, Miller BF, Evans VM, Warren JS. The membrane attack complex of complement induces interleukin-8 and monocyte chemoattractant protein-1 secretion from human umbilical vein endothelial cells. Am J Pathol. 1996;149(3):953-961.

26. Smith JD, Yacoub MH, Rose ML. Endothelial cell activation by sera containing HLA antibodies is mediated by interleukin-1. Transplantation. 1998;66(9):1229-1237.

27. Heeger PS, et al. Pretransplant frequency of donor-specific, IFN-gamma-producing lymphocytes is a manifestation of immunologic memory and correlates with the risk of posttransplant rejection episodes. J Immunol. 1999;163(4):2267-2275.

28. Kärre K, Ljunggren HG, Piontek G, Kiessling R. Selective rejection of H-2-deficient lymphoma variants suggests alternative immune defence strategy. Nature. 1986;319(6055):675-678.

29. Pende D, et al. PVR (CD155) and Nectin-2 (CD112) as ligands of the human DNAM-1 (CD226) activating receptor: involvement in tumor cell lysis. Mol Immunol. 2005;42(4):463-469.

30. Stanietsky N, et al. The interaction of TIGIT with PVR and PVRL2 inhibits human NK cell cytotoxicity. Proc Natl Acad Sci USA. 2009;106(42):17858-17863.

31. Béziat V, Hervier B, Achour A, Boutolleau D, Marfain-Koka A, Vieillard V. Human NKG2A overrides NKG2C effector functions to prevent autoreactivity of NK cells. Blood. 2011;117(16):4394-4396.

32. Schechner JS, et al. In vivo formation of complex microvessels lined by human endothelial cells in an immunodeficient mouse. Proc Natl Acad Sci USA. 2000;97(16):9191-9196.

33. Zheng L, Gibson TF, Schechner JS, Pober JS, Bothwell AL. Bcl-2 transduction protects human endothelial cell synthetic microvessel grafts from allogeneic T cells in vivo. J Immunol. 2004;173(5):3020-3026.

34. Suárez Y, Shepherd BR, Rao DA, Pober JS. Alloimmunity to human endothelial cells derived from cord blood progenitors J Immunol. 2007;179(11):7488-7496.

35. Qin L, et al. Complement C5 inhibition reduces T cell-mediated allograft vasculopathy caused by both alloantibody and ischemia reperfusion injury in humanized mice. Am J Transplant. 2016;16(10):2865-2876.

36. Briscoe DM, et al. The allogeneic response to cultured human skin equivalent in the hu-PBL-SCID mouse model of skin rejection. Transplantation. 1999;67(12):1590-1599.

37. Lechler RI, Batchelor JR. Restoration of immunogenicity to passenger cell-depleted kidney allografts by the addition of donor strain dendritic cells. J Exp Med. 1982;155(1):31-41.

38. Brewer Y, et al. Effect of graft perfusion with two CD45 monoclonal antibodies on incidence of kidney allograft rejection. Lancet. 1989;2(8669):935-937.

39. Appay V, et al. Characterization of CD4(+) CTLs ex vivo. J Immunol. 2002;168(11):5954-5958.

40. Giaretta F, et al. Different regulatory and cytotoxic $\mathrm{CD}^{+} \mathrm{T}$ lymphocyte profiles in renal transplants with antibody-mediated chronic rejection or long-term good graft function. Transpl Immunol. 2013;28(1):48-56.

41. Zhang Q, Reed EF. The importance of non-HLA antibodies in transplantation. Nat Rev Nephrol. 2016;12(8):484-495.

42. Das K, et al. Generation of murine tumor cell lines deficient in MHC molecule surface expression using the CRISPR/Cas9 system. PLoS One. 2017;12(3):e0174077.

43. Mandal PK, et al. Efficient ablation of genes in human hematopoietic stem and effector cells using CRISPR/Cas9. Cell Stem Cell. 2014;15(5):643-652.

44. Ardolino M, et al. Cytokine therapy reverses NK cell anergy in MHC-deficient tumors. J Clin Invest. 2014;124(11):4781-4794.

45. Bender JR, Pardi R, Engleman E. T-cell receptor-negative natural killer cells display antigen-specific cytotoxicity for microvascular endothelial cells. Proc Natl Acad Sci USA. 1990;87(18):6949-6953.

46. Pietra BA, Wiseman A, Bolwerk A, Rizeq M, Gill RG. CD4 T cell-mediated cardiac allograft rejection requires donor but not host MHC class II. J Clin Invest. 2000;106(8):1003-1010.

47. Gorer PA, Lyman S, Snell GD. Studies on the genetic and antigenic basis of tumour transplantation - linkage between a histocompatibility gene and fused in mice. Proc Royal Soc B. 1948;135(881):499-505.

48. Rongvaux A, et al. Development and function of human innate immune cells in a humanized mouse model. Nat Biotechnol. 2014;32(4):364-372

49. Banno K, Yoder MC. Tissue regeneration using endothelial colony-forming cells: promising cells for vascular repair. Pediatr Res. 2018;83(1-2):283-290

50. Basile DP, Yoder MC. Circulating and tissue resident endothelial progenitor cells. J Cell Physiol. 2014;229(1):10-16.

51. Jang Y, et al. Development of immunocompatible pluripotent stem cells via CRISPR-based human leukocyte antigen engineering. Exp Mol Med. 2019;51(1):3.

52. Gutierrez-Aranda I, et al. Human induced pluripotent stem cells develop teratoma more efficiently and faster than human embryonic stem cells regardless the site of injection. Stem Cells. 2010;28(9):1568-1570.

53. Kim SW, et al. Therapeutic effects of late outgrowth endothelial progenitor cells or mesenchymal stem cells derived from human umbilical cord blood on infarct repair. Int J Cardiol. 2016;203:498-507.

54. Moubarik C, et al. Transplanted late outgrowth endothelial progenitor cells as cell therapy product for stroke. Stem Cell Rev Rep. 2011;7(1):208-220.

55. Medina RJ, O'Neill CL, Humphreys MW, Gardiner TA, Stitt AW. Outgrowth endothelial cells: characterization and their potential for reversing ischemic retinopathy. Invest Ophthalmol Vis Sci. 2010;51(11):5906-5913.

56. Wang $\mathrm{CH}$, et al. Late-outgrowth endothelial cells attenuate intimal hyperplasia contributed by mesenchymal stem cells after 
vascular injury. Arterioscler Thromb Vasc Biol. 2008;28(1):54-60.

57. Melero-Martin JM, De Obaldia ME, Allen P, Dudley AC, Klagsbrun M, Bischoff J. Host myeloid cells are necessary for creating bioengineered human vascular networks in vivo. Tissue Eng Part A. 2010;16(8):2457-2466.

58. Joo HJ, et al. Smooth muscle progenitor cells from peripheral blood promote the neovascularization of endothelial colony-forming cells. Biochem Biophys Res Commun. 2014;449(4):405-411.

59. Coppens V, et al. Human blood outgrowth endothelial cells improve islet survival and function when co-transplanted in a mouse model of diabetes. Diabetologia. 2013;56(2):382-390.

60. Lin Y, Chang L, Solovey A, Healey JF, Lollar P, Hebbel RP. Use of blood outgrowth endothelial cells for gene therapy for hemophilia A. Blood. 2002;99(2):457-462.

61. Lin RZ, et al. Induction of erythropoiesis using human vascular networks genetically engineered for controlled erythropoietin release. Blood. 2011;118(20):5420-5428.

62. Wang HW, et al. Human umbilical cord mesenchymal stem cells derived from Wharton's jelly differentiate into insulin-producing cells in vitro. Chin Med J. 2011;124(10):1534-1539.

63. Shepherd BR, Enis DR, Wang F, Suarez Y, Pober JS, Schechner JS. Vascularization and engraftment of a human skin substitute using circulating progenitor cell-derived endothelial cells. FASEB J. 2006;20(10):1739-1741.

64. Trapnell C, Pachter L, Salzberg SL. TopHat: discovering splice junctions with RNA-Seq. Bioinformatics. 2009;25(9):1105-1111.

65. Trapnell C, et al. Transcript assembly and quantification by RNA-Seq reveals unannotated transcripts and isoform switching during cell differentiation. Nat Biotechnol. 2010;28(5):511-515.

66. Benjamini Y, Drai D, Elmer G, Kafkafi N, Golani I. Controlling the false discovery rate in behavior genetics research. Behav Brain Res. 2001;125(1-2):279-284.

67. Pierce RW, et al. A p190BRhoGAP mutation and prolonged RhoB activation in fatal systemic capillary leak syndrome. $J$ Exp Med. 2017;214(12):3497-3505 